\title{
Computational modeling of fully ionized magnetized plasmas using the fluid approximation ${ }^{a)}$
}

\author{
D. D. Schnack ${ }^{\text {b) }}$ \\ Center for Energy and Space Science, Science Applications International Corporation, \\ 10260 Campus Point Drive, San Diego, California 92121 \\ D. C. Barnes \\ Center for Integrated Plasma Studies, University of Colorado, 2000 Colorado Avenue, Boulder, \\ Colorado 80309 \\ D. P. Brennan \\ General Atomics, P.O. Box 85608, San Diego, California 92186 \\ C. C. Hegna \\ Department of Engineering Physics, University of Wisconsin, 1500 Engineering Drive, Madison, \\ Wisconsin 53706 \\ E. Held \\ Department of Physics, Utah State University, Logan, Utah 84322 \\ C. C. Kim \\ Department of Engineering Physics, University of Wisconsin, 1500 Engineering Drive, Madison, \\ Wisconsin 53706 and Plasma Science and Innovation Center, University of Washington, P.O. Box 352250, \\ Seattle, Washington 98195 \\ S. E. Kruger \\ TechX Corporation, 5621 Arapahoe Avenue, Suite A, Boulder, Colorado 80303
}

A. Y. Pankin

Center for Energy and Space Science, Science Applications International Corporation, 10260 Campus Point Drive, San Diego, California 92121

C. R. Sovinec

Department of Engineering Physics, University of Wisconsin, 1500 Engineering Drive, Madison, Wisconsin 53706

(Received 20 October 2005; accepted 6 January 2006; published online 11 May 2006)

\begin{abstract}
Strongly magnetized plasmas are rich in spatial and temporal scales, making a computational approach useful for studying these systems. The most accurate model of a magnetized plasma is based on a kinetic equation that describes the evolution of the distribution function for each species in six-dimensional phase space. High dimensionality renders this approach impractical for computations for long time scales. Fluid models are an approximation to the kinetic model. The reduced dimensionality allows a wider range of spatial and/or temporal scales to be explored. Computational modeling requires understanding the ordering and closure approximations, the fundamental waves supported by the equations, and the numerical properties of the discretization scheme. Several ordering and closure schemes are reviewed and discussed, as are their normal modes, and algorithms that can be applied to obtain a numerical solution. (C) 2006 American Institute of Physics. [DOI: 10.1063/1.2183738]
\end{abstract}

\section{INTRODUCTION}

Mathematical models of dynamical systems, such as strongly magnetized plasmas, contain widely separated space and time scales. The complexity of these systems generally dictates that a computational approach is the only practical theoretical means of obtaining insight into their behavior. Often, the dynamics of interest occur on an intermediate time scale between the slowest and fastest characteristic frequencies of the system. The highest frequencies are parasitic

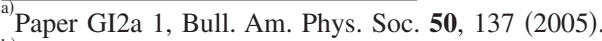

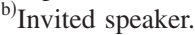

waves that may be of secondary interest to the dominant dynamics, but that can nonetheless inhibit the efficiency of numerical algorithms. Accurate and efficient calculation of the dynamics of low frequency motions in the presence of high frequency parasitic modes is among the major challenges of computational physics.

One approach to solving this problem is to employ an analytic reduction of the primitive mathematical model with the aim of eliminating the offending parasitic modes before the computation begins. A simple and well known example of such a reduction is the assumption of incompressibility in fluid mechanics. This eliminates high frequency sound waves 
from the system and allows the calculation to proceed at a rate determined by the large scale bulk flows, which presumably contain the interesting dynamics. (The time step can be increased by a factor of the ratio of the sound speed to the flow velocity, which is a significant improvement for subsonic flows.)

The magnetohydrodynamic (MHD) model commonly employed to analyze magnetic fusion plasmas contains several fast time scales, most of which have little or no effect on the lower frequency motions. Reduced models have been developed for these systems ${ }^{1-4}$ that make various assumptions about the magnetic geometry, the relative strengths of the fluid and magnetic pressures, the relative strengths of various magnetic field components, and the degree to which the system is constrained to obey equilibrium force balance. Sometimes they explicitly take advantage of approximate cancellations of large terms that can occur in certain parameter regimes. $^{5-13}$

Reduced models often use potential representations of vector fields to recast the equations in terms of scalar variables, and this approach can be extended to include all the physics contained in the primitive model. One advantage of this approach is to facilitate the approximate cancellation of large terms that may occur under certain restricted orderings of the fluid variables (see Sec. II F). The resulting equations often appear to be more complicated and less physically intuitive than the original primitive formulation. Nonetheless, these formulations can provide insights into some aspects of plasma dynamics, and they often form the starting point for analytical investigation.

An alternative approach is to retain the primitive form and variables of the mathematical model, and to use methods of advanced computational physics to mitigate the effects of the parasitic modes. ${ }^{14-28}$ These usually employ strongly implicit time advance algorithms, and are able to advance the solution accurately and stably over very large time steps. The advantage is that all the underlying physics is retained, the model remains valid across a wide range of parameters, the boundary conditions are obvious, and the same algorithm can be applied to a wide variety of problems pertaining to magnetized plasmas. A disadvantage is that it becomes more difficult to make direct comparison with various analytical studies, since these often use reduced models.

We have found the primitive models to be advantageous in many cases because of their versatility and relative ease of implementation. In this paper, we describe the extension of this approach to nonlinear simulations of the extended MHD equations, including Hall, electron diamagnetic, and finite ion Larmor radius (FLR) effects in a variety of realistic magneto-plasma configurations. The requirements of these calculations have stretched the limits of present computational hardware and software performance.

The extended MHD model is appropriate for describing many of the interacting physical processes that occur in hot, magnetized plasmas. In order to properly design algorithms for the solution of the primitive form of the extended MHD equations, and to interpret the results, it is important to understand the parameter ranges in which various terms are important, and how these various terms relate to reduced models that are common in the literature. We therefore begin with a review of several of the models that can be deduced from primitive extended MHD, both to illustrate the richness of the overall model and to elucidate the utility of the various subsidiary models that follow from the proper ordering of terms. We pay special attention to the wave dispersion properties of these models, as these can strongly affect the design of algorithms. In the end, of course, we solve the primitive equations using advanced computational techniques to eliminate or highlight various properties of the model.

This paper is organized as follows. In Sec. II we present the nondimensional two-fluid and FLR equations for force balance, and introduce the parameters of the model and their ordering. Various issues related to the stress tensor are also discussed. We show how the Hall, ideal, and drift MHD regimes of extended MHD can be deduced by a systematic ordering of the terms in the general model. In Sec. III we investigate the wave dispersion and stability properties of the extended MHD model, and show that it introduces dispersive modifications to the ideal MHD spectrum. We describe the effect of the two-fluid and FLR terms in the case of the simple gravitational interchange instability. We also briefly address the role that the ion heat stress terms in the gyroviscosity may play in both wave dispersion and stability, and show that its effects are expected to be negligible. In Sec. IV we describe some algorithms for obtaining a numerical solution of the extended MHD equations. Techniques useful for resistive MHD are briefly reviewed. However, we concentrate on some new approaches that address the modified dispersive properties of the extended MHD model. In Sec. V we address the difficult topic of kinetic closures of the fluid equations. Parallel closures are nonlocal formulations for the electron parallel heat flux and ion parallel collisional stress tensor. Closures that determine modifications to the stress tensor as a result of an energetic minority ion species can be computed by subcycling a set of kinetic equations and evaluating the required moments numerically. We demonstrate that efficient and stable algorithms to compute these effects can be incorporated into the general extended MHD model. Our experience in these problems is briefly summarized in Sec. VI.

\section{THE EXTENDED MHD MODEL}

The familiar MHD model describes perpendicular dynamics of a continuous electrically conducting fluid medium permeated by a magnetic field. The equations of the model can be derived without recourse to kinetic theory. ${ }^{31}$ A basic assumption of the model is that all physical quantities are "averaged over elements of volume that are "physically infinitesimal,' ignoring the microscopic variations...that result from the molecular structure of matter." 29 In a plasma, this model cannot describe effects due to the cyclotron gyration of individual charged particles in a magnetic field (so called FLR, or finite Larmor radius, effects ${ }^{5,30}$ ), or the average drift of these orbits relative to the magnetic field. Further, in a hot magnetized plasma, the particle mean free path parallel to the magnetic field often exceeds all other macroscopic scale lengths, thus negating the basic MHD assumption and dictat- 
ing a kinetic treatment. For these parameter regimes, one must apply a fluid theory rigorously derived from the moments of the underlying kinetic equation. ${ }^{5,13}$ This procedure produces a hierarchy of equations for successively higher velocity moments of the distribution function. In principle, this infinite set of equations is equivalent to the kinetic description. In practice, the set must be truncated at some order by an independent expression (closure relation) for the highest order velocity moment. The resulting set of equations contains several nondimensional parameters whose relative ordering (i.e., large or small) can further simplify the model and isolate specific physical effects.

The nondimensional parameters that characterize the fluid equations are $\varepsilon=\omega / \Omega_{i}$, the ratio of the characteristic frequency to the ion gyro-frequency, $\xi=V_{0} / V_{t h i}$, the ratio of the characteristic flow velocity to the ion thermal speed, $\delta$ $=\rho_{i} / L$, the ratio of the ion Larmor (gyro-) radius to the macroscopic scale length, $\nu / \boldsymbol{\Omega}_{i}$, the ratio of the collision frequency to the ion gyro-frequency, and $\beta_{i}=\left(V_{t h i} / V_{A}\right)^{2}$, the square of the ratio of the ion thermal speed to the Alfvén speed. In a magnetized plasma, one parameter, $\delta$, can be considered small. The other parameters are ordered large or small relative to $\delta$. The resulting equations will have terms that are proportional to various powers of $\delta$. By systematically ignoring terms that are smaller than some order of $\delta$, we will arrive at a hierarchy of fluid models that are valid for describing different types of plasma dynamics. We refer to these models collectively as extended MHD.

Computational implementation of extended MHD models is complicated by the dispersive modes that are introduced by two-fluid and FLR effects. Whistler waves are eliminated analytically from both the MHD and drift models by the constraints of force balance. However, in the drift model the dispersive kinetic Alfvén wave ${ }^{31}$ (KAW) remains. The KAW modes are described by a wave equation that is second order in time and fourth order in space, and is quite similar in structure to the Whistler wave equation. In general, both sets of modes appear in the model. A similar situation arises with the inclusion of the gyro-viscous stress in the ion equation of motion. This introduces dispersive modifications to the Alfvén waves.

The dispersive modifications introduced by the extended MHD model require special attention in the design of numerical algorithms. Explicit time advance methods result in unacceptable time step limitations. Implicit methods require the inversion of operators that have no counterpart in ideal or resistive MHD, and present challenges to the design of linear solver software. These properties combine to make the numerical solution of the nonlinear extended MHD model in realistic geometry one of the more difficult problems in computational physics.

\section{A. Ordered fluid equations}

Before attempting to understand any numerical solution of the fluid equations, it is essential to understand the basic limitations and properties of the model being used. The fluid moment equations are a shortcut to obtaining an approximate solution of the kinetic equation. Several interesting proper- ties of the fluid equations can be illustrated by considering only the lowest (zeroth and first) order velocity moments of the kinetic equation. Of course, equations for higher order moments (e.g., energy and heat flow) are required to describe the thermodynamics, but issues relating to mass conservation and force balance are sufficient to elucidate several of the important issues.

This goal is facilitated by writing the extended MHD equations in nondimensional form. Assuming quasineutrality and neglecting the electron mass, the continuity and momentum balance equations for ions and electrons are

$$
\begin{aligned}
\varepsilon \frac{\partial n}{\partial t}=-\xi \delta \nabla \cdot n \mathbf{V}_{i}= & -\xi \delta \nabla \cdot n \mathbf{V}_{e}, \\
\varepsilon \xi \frac{\partial \mathbf{V}_{i}}{\partial t}+\xi^{2} \delta \mathbf{V}_{i} \cdot \nabla \mathbf{V}_{i}= & -\frac{1}{n} \delta\left(\nabla p_{i}+\frac{\Pi_{i 0}}{p_{0}} \nabla \cdot \Pi_{i}\right) \\
& +\xi\left(\mathbf{E}+\mathbf{V}_{i} \times \mathbf{B}\right),
\end{aligned}
$$

and

$$
\xi \mathbf{E}=-\xi \mathbf{V}_{e} \times \mathbf{B}-\frac{1}{n} \delta\left(\nabla p_{e}+\frac{\Pi_{e 0}}{p_{0}} \nabla \cdot \Pi_{e}\right) .
$$

The friction force has been neglected for simplicity, and it is assumed that there exist independent expressions or equations for the scalar pressures $p_{\alpha}$ and the stress tensors $\Pi_{\alpha}$. The stress tensor will be discussed further in Sec. II B. The nondimensional "pre-Maxwell" equations are

$$
\varepsilon \frac{\partial \mathbf{B}}{\partial t}=-\xi \delta \nabla \times \mathbf{E}
$$

and

$$
\mathbf{J}=\xi \nabla \times \mathbf{B},
$$

along with the constitutive relation

$$
\mathbf{J}=n\left(\mathbf{V}_{i}-\mathbf{V}_{e}\right) .
$$

In Eqs. (1c), (2a), and (3), the velocity is measured in units of $V_{0}(\mathrm{~m} / \mathrm{s})$, the magnetic field is measured in units of $B_{0}(\mathrm{~T})$, the density in units of $n_{0}\left(\mathrm{~m}^{-3}\right)$, the electric field in units of $E_{0}=V_{0} B_{0}(\mathrm{~V} / \mathrm{m})$, the current density in units of $J_{0}$ $=n_{0} e V_{0}\left(\mathrm{Amp} / \mathrm{m}^{2}\right)$, pressure is measured in units of $m_{i} n_{0} V_{t h i}^{2}$ (Pa, where $V_{t h i}=\sqrt{2 T_{i} / m_{i}}$ is the ion thermal speed), distance in units of $L \sim \nabla_{\perp}^{-1}(\mathrm{~m})$, and time in units of $t_{0}=\omega^{-1}(\mathrm{~s})$, where $\omega$ is some characteristic frequency. This normalization allows the relative units of the electric field and current density to vary with $V_{0}$, thus keeping the nondimensional dependent variables in scale. The stress tensor is measured in units of $\Pi_{\alpha 0}(\mathrm{~Pa})$; choices for this factor will be discussed in Sec. II B. The dimensionless constants appearing as coefficients in Eqs. (1c), (2a), and (3) are $\varepsilon=\omega / \Omega, \xi=V_{0} / V_{\text {thi }}$, and $\delta$ $=\rho_{i} / L$, where $\Omega=e B_{0} / m$ is the ion cyclotron frequency and $\rho_{i}=V_{t h i} / \Omega$ is the ion Larmor radius.

The ion and electron fluid velocities can be decomposed into perpendicular and parallel parts as $\mathbf{V}_{\alpha}=\mathbf{V}_{\perp \alpha}+\mathbf{V}_{\| \alpha}$. From Eqs. (1c) and (1d), the perpendicular parts can be expressed as 


$$
\begin{aligned}
\xi \mathbf{V}_{\perp i}= & \xi \frac{\mathbf{E} \times \mathbf{B}}{B^{2}}+\delta \frac{1}{n B^{2}} \mathbf{B} \times\left(\nabla p_{i}+\frac{\Pi_{i 0}}{p_{0}} \nabla \cdot \Pi_{i}\right)+\xi \frac{1}{B^{2}} \mathbf{B} \\
& \times\left(\varepsilon \frac{\partial \mathbf{V}_{i}}{\partial t}+\xi \delta \mathbf{V}_{i} \cdot \nabla \mathbf{V}_{i}\right)
\end{aligned}
$$

and

$$
\xi \mathbf{V}_{\perp e}=\xi \frac{\mathbf{E} \times \mathbf{B}}{B^{2}}-\delta \frac{1}{n B^{2}} \mathbf{B} \times\left(\nabla p_{e}+\frac{\Pi_{e 0}}{p_{0}} \nabla \cdot \Pi_{e}\right)
$$

The perpendicular ion and electron velocities have a common part, $\mathbf{V}_{E}=\mathbf{E} \times \mathbf{B} / B^{2}$. (This is called the MHD velocity.) The determination of the parallel dynamics may require a kinetic theory. yield

Finally, Eqs. (1c), (1d), and (3) may be combined to

$n\left(\varepsilon \xi \frac{\partial \mathbf{V}_{i}}{\partial t}+\xi^{2} \delta \mathbf{V}_{i} \cdot \nabla \mathbf{V}_{i}\right)=\xi \mathbf{J} \times \mathbf{B}-\frac{1}{n} \delta\left(\nabla p+\frac{\Pi_{i 0}}{p_{0}} \nabla \cdot \Pi_{i}\right)$

This is the equation of motion for the momentum carrying component of the fluid plasma. In Eq. (5), we have set $p$ $=p_{e}+p_{i}$. We can also substitute Eq. (3) into Eq. (1d) to obtain the generalized Ohm's law

$$
\xi \mathbf{E}=-\xi \mathbf{V}_{i} \times \mathbf{B}+\xi \frac{1}{n} \mathbf{J} \times \mathbf{B}-\delta_{n}^{-\frac{1}{n}}\left(\nabla p_{e}+\frac{\Pi_{e 0}}{p_{0}} \nabla \cdot \Pi_{e}\right) .
$$

Note that the terms $\mathbf{V}_{i} \times \mathbf{B}$ and $\mathbf{J} \times \mathbf{B}$ appear formally at the same order as $\mathbf{E}$.

The importance of the various terms in Eqs. (1c), (2a), (3), (4a), (5), and (6) is determined by the relative sizes of the dimensionless constants $\delta$, $\varepsilon$, and $\xi$. For the strongly magnetized plasma considered here, we can always assume $\delta \ll 1$. Different fluid models emerge depending on how we order the remaining dimensionless variables with respect to $\delta$.

\section{B. The stress tensor}

Further progress requires some knowledge of the ion stress tensor $\Pi_{i}$. A well known formulation is that given by Braginskii, ${ }^{32}$ which assumes small parallel mean free path, small Larmor radius, and collision frequency large compared with the characteristic frequency. The stress tensor can be written in terms of its parallel $(\mathbf{b b})$, cross $(\mathbf{b} \times \mathbf{I})$, and perpendicular (I-bb) components as

$$
\Pi_{i}=\Pi_{\|}+\Pi_{\curlywedge}+\Pi_{\perp},
$$

where

$$
\begin{aligned}
& \Pi_{\|}=-\frac{3}{2} \eta_{0}(\mathbf{b} \cdot \mathbf{W} \cdot \mathbf{b})\left(\mathbf{I}-\frac{1}{3} \mathbf{b b}\right), \\
& \Pi_{\curlywedge}=\frac{\eta_{3}}{2}[\mathbf{b} \times \mathbf{W} \cdot(\mathbf{I}+3 \mathbf{b} \mathbf{b})+\text { transpose }],
\end{aligned}
$$

$$
\begin{aligned}
\Pi_{\perp}= & -\eta_{1}\left\{(\mathbf{I}-\mathbf{b b}) \cdot \mathbf{W} \cdot(\mathbf{I}-\mathbf{b b})-\frac{1}{2}(\mathbf{I}-\mathbf{b b})(\mathbf{I}-\mathbf{b b}): \mathbf{W}\right. \\
& +4[(\mathbf{I}-\mathbf{b b}) \cdot \mathbf{W} \cdot \mathbf{b b}+\text { transpose }]\}
\end{aligned}
$$

$$
\mathbf{W}=\nabla \mathbf{V}_{i}+\nabla \mathbf{V}_{i}^{T}-\frac{2}{3} \mathrm{I} \nabla \cdot \mathbf{V}_{i}
$$

and

$$
\begin{aligned}
& \eta_{0}=0.96 \frac{n T_{i}}{\nu}, \\
& \eta_{1}=\frac{3}{10} \frac{n T_{i} \nu}{\Omega^{2}}, \\
& \eta_{3}=\frac{n T_{i}}{2 \Omega},
\end{aligned}
$$

where $\nu$ is the collision frequency. Equations (8a)-(8d) express the stress tensor in terms of gradients of the velocity, and therefore close the system of equations.

The stress tensor can be written in nondimensional form using the normalization given in Sec. II A. The result is

$$
\frac{\Pi_{i 0}}{p_{i 0}} \nabla \cdot \Pi_{i}=\xi \delta\left[\frac{1}{\nu / \Omega} \nabla \cdot \Pi_{\|}+\nabla \cdot \Pi_{\mathrm{gv}}+\frac{\nu}{\Omega} \nabla \cdot \Pi_{\perp}\right\rfloor .
$$

The classical Braginskii expression for the parallel viscous force, which decreases with increasing collisionality, is not valid in a hot confined plasma such as a tokamak. Instead, this force is determined from neoclassical theory, ${ }^{33}$ which provides expressions for the parallel force for all collisionality regimes. A local expression for this force can be approximated as ${ }^{34}$

$$
\nabla \cdot \Pi_{\|}=\nabla \cdot \Pi_{i}^{\mathrm{nc}}=m n\left\langle B^{2}\right\rangle \mu_{i} \frac{V_{\theta i}}{B_{\theta}^{2}} \mathbf{e}_{\theta},
$$

where $\mu_{i}$ is the neoclassical damping coefficient and $\mathbf{e}_{\theta}$ is a unit vector in the poloidal direction (see Sec. V C). Introducing the dimensionless variables of Sec. II A into this expression, we find the normalization

$$
\frac{\Pi_{0}^{n c}}{p_{0}}=\frac{\xi}{\delta} \frac{\mu}{\Omega}
$$

The so-called "banana regime" that is of interest in a low collisionality tokamak occurs when $\nu / \Omega \ll \varepsilon_{A}^{3 / 2}\left(\omega_{b} / \Omega\right)$ $\sim \varepsilon_{A}^{3 / 2} \delta / q$, where $\varepsilon_{A}$ is the inverse aspect ratio of the device, $\omega_{b}$ is the bounce frequency, and $q$ is the local safety factor. It is therefore consistent to order $\nu / \Omega \sim \delta^{2}$. In this regime, neoclassical theory shows that $\mu \sim \varepsilon_{A}^{1 / 2} \nu$, so that Eq. (12) becomes

$$
\frac{\Pi_{0}^{\mathrm{nc}}}{p_{0}}=\frac{\xi}{\delta} \varepsilon_{A}^{1 / 2} \frac{\nu}{\Omega} \sim \varepsilon_{A}^{1 / 2} \xi \delta .
$$

Despite the previous discussion, it is common in nonlinear computational models to introduce an isotropic artificial viscosity of the form 


$$
\frac{\Pi_{0}^{\mathrm{visc}}}{p_{0}} \nabla \cdot \Pi^{\mathrm{visc}}=-n \mu_{A} \nabla^{2} \mathbf{V} .
$$

This expression resembles the zero field limit of the Braginskii model, and is not valid for a highly magnetized plasma. Further, it does not vanish for rigid rotational motions. [A more appropriate, although more complicated, expression would be $\nabla \cdot n \mu_{A} \mathbf{W}$, where $\mathbf{W}$ is given by Eq. (8d).] However, it is relatively simple to implement and is widely used nonetheless. We will consider the artificial viscosity coefficient $\mu_{A}$ to be of adjustable order in $\delta$, meaning that we can choose its order so that it appears wherever we wish in the final equations.

With these caveats, the nondimensional viscous force in the banana regime is

$$
\begin{aligned}
\frac{\Pi_{i 0}}{p_{0}} \nabla \cdot \Pi_{i}= & -n \mu_{A} \nabla^{2} \mathbf{V}_{i}+\xi \delta\left[\nabla \cdot \Pi_{i}^{\mathrm{gv}}+\varepsilon_{A}^{1 / 2} \nabla \cdot \Pi_{i}^{n c}\right. \\
& \left.+\delta^{2} \nabla \cdot \Pi_{\perp i}\right] .
\end{aligned}
$$

The Braginskii expression for the electron viscous force is proportional to the electron mass, and is therefore ignored when it appears in concert with the ion stress. However, the parallel viscous stress for the electrons will be taken to be neoclassical.

For reference, it is instructive to write down the full momentum balance equation, including the viscous stress, without any ordering assumptions. The result is

$$
\begin{aligned}
\xi \mathbf{J} \times \mathbf{B}-\delta \nabla p= & n\left(\varepsilon \xi \frac{\partial \mathbf{V}_{i}}{\partial t}+\xi^{2} \delta \mathbf{V}_{i} \cdot \nabla \mathbf{V}_{i}\right)+\xi \delta^{2}\left(\frac{1}{\nu / \Omega} \nabla \cdot \Pi_{\|}\right. \\
& \left.+\nabla \cdot \Pi_{i}^{\mathrm{gv}}+\frac{\nu}{\Omega} \nabla \cdot \Pi_{\perp}\right)+\xi \frac{\mu}{\Omega} \nabla \cdot \Pi_{i}^{n c} \\
& -n \mu_{A} \nabla^{2} \mathbf{V}_{i} .
\end{aligned}
$$

The terms on the left hand side contain the "equilibrium" forces. The terms on the right hand side are all proportional to the velocity and are the plasma response when the system deviates from force balance. As stated following Eq. (14), the artificial viscous parameter $\mu_{A}$ is adjustable with respect to $\delta$ and will be chosen for convenience in the later sections.

We remark that, for low collisionality, Eq. (8b) for the gyro-viscous stress should contain the additional term ${ }^{35-37}$

$$
\Pi_{\wedge_{q}}=\frac{2}{5 \Omega}\left[\mathbf{b} \times \mathbf{W}_{q} \cdot(\mathbf{I}+3 \mathbf{b b})+\text { transpose }\right],
$$

where

$$
\mathbf{W}_{q}=\nabla \mathbf{q}_{i}+\nabla \mathbf{q}_{i}^{T}-\frac{2}{3} \mathbf{I} \nabla \cdot \mathbf{q}_{i},
$$

where $\mathbf{q}_{i}$ is the ion heat stress. The effect of these terms will be discussed in Sec. III D.

\section{The Hall MHD ordering}

For generality of application, we would like to permit flows of arbitrary speeds and large accelerations, as might occur, for example, in shock tubes, fast Z-pinches, fast opening switches, and the inner heliosphere. We are thus led to order the frequency and flow as

$$
\varepsilon \sim 1, \quad \xi \sim 1 / \delta .
$$

Neither the Braginskii nor the neoclassical closures for the parallel ions stress were derived for this regime, and we ignore them for now. Then, the continuity, ion, and electron equations become

$$
\begin{aligned}
& \frac{\partial n}{\partial t}=-\nabla \cdot n \mathbf{V}_{i}, \\
& \mathbf{J} \times \mathbf{B}=n \frac{d \mathbf{V}_{i}}{d t}+\frac{1}{n} \delta^{2}\left(\nabla p+\nabla \cdot \Pi_{\mathrm{gv}}\right)+O\left(\delta^{3}\right)
\end{aligned}
$$

(where $\left.d / d t=\partial / \partial t+\mathbf{V}_{i} \cdot \nabla\right)$, and

$$
\mathbf{E}=-\mathbf{V}_{e} \times \mathbf{B}-\frac{1}{n} \delta^{2} \nabla p_{e},
$$

along with

$$
\begin{aligned}
& \delta \mathbf{J}=\nabla \times \mathbf{B}, \\
& \frac{\partial \mathbf{B}}{\partial t}=-\nabla \times \mathbf{E},
\end{aligned}
$$

and

$$
\mathbf{J}=n\left(\mathbf{V}_{i}-\mathbf{V}_{e}\right) .
$$

From Eq. (20b), we see that there is force imbalance at $O(1)$. This allows for very fast flows and rapid acceleration. The lowest order correction is from the parallel viscous and neoclassical forces. Pressure and gyro-viscous forces enter at $O\left(\delta^{2}\right)$. In this model, the dynamics are dominated by the Lorentz force. The electron velocity is $\mathbf{V}_{e}=\mathbf{V}_{i}-\mathbf{J} / n$, so that the ions and electrons flow as separate fluids, and Ohm's law must now be written as

$$
\mathbf{E}=-\mathbf{V}_{i} \times \mathbf{B}+\frac{1}{n} \mathbf{J} \times \mathbf{B}+O\left(\delta^{2}\right)
$$

Since $\mathbf{J} \times \mathbf{B} \sim O(1)$ [see Eq. (20b)], these dynamics enter at lowest order and must be retained to describe very fast flows, thus the terminology Hall MHD.

In the banana collisionality regime $\left(\nu / \Omega_{i} \sim \mu / \Omega \sim \delta^{2}\right)$, Eq. (20b) becomes

$$
\begin{aligned}
\mathbf{J} \times \mathbf{B}= & n \frac{d \mathbf{V}_{i}}{d t}+\nabla \cdot \Pi_{\|}+\delta \nabla \cdot \Pi_{i}^{\mathrm{nc}}+\frac{1}{n} \delta^{2}\left(\nabla p+\nabla \cdot \Pi_{\mathrm{gv}}\right) \\
& +O\left(\delta^{4}\right) .
\end{aligned}
$$

The promotion of the parallel viscous stress to $O(1)$ is unphysical, since neither the Braginskii nor neoclassical closures were derived under these conditions. They can be replaced by the artificial isotropic viscous stress at $O(\delta)$. 


\section{The MHD ordering}

Supersonic flows rarely occur in a confined plasma, so for such cases we can restrict the flows to be on the order of the thermal velocity, and require low frequencies (compared with the cyclotron frequency). We thus define the MHD ordering $^{13}$ as

$$
\varepsilon \sim \delta, \quad \xi \sim 1
$$

The relevant equations are then

$$
\begin{aligned}
& \frac{\partial n}{\partial t}+\nabla \cdot n \mathbf{V}_{i}=0, \\
& n \delta \frac{d \mathbf{V}_{i}}{d t}=\mathbf{J} \times \mathbf{B}-\delta\left(\nabla p+\frac{\Pi_{i 0}}{p_{0}} \nabla \cdot \Pi_{i}\right), \\
& \mathbf{E}=-\mathbf{V}_{e} \times \mathbf{B}-\delta \frac{1}{n}\left(\nabla p_{e}+\frac{\Pi_{e 0}}{p_{0}} \nabla \cdot \Pi_{e}\right), \\
& \frac{\partial \mathbf{B}}{\partial t}=-\nabla \times \mathbf{E},
\end{aligned}
$$

and

$$
\mathbf{J}=\nabla \times \mathbf{B},
$$

where $d / d t=\partial / \partial t+\mathbf{V}_{i} \cdot \nabla$. In the plateau regime the momentum balance equation becomes

$$
\begin{aligned}
\mathbf{J} \times \mathbf{B}= & \delta\left(n \frac{d \mathbf{V}_{i}}{d t}+\nabla p+\nabla \cdot \Pi_{\|}+\nabla \cdot \Pi_{i}^{\mathrm{nc}}\right)+\delta^{2} \nabla \cdot \Pi_{i}^{\mathrm{gv}} \\
& +O\left(\delta^{4}\right) .
\end{aligned}
$$

Deviations from a force-free $(\mathbf{J} \times \mathbf{B}=0)$ state now only appear at $O(\delta)$. Both the Braginskii and neoclassical viscous stresses appear formally at this order. The electron equation is

$$
\mathbf{E}=-\mathbf{V}_{e} \times \mathbf{B}+O(\delta) .
$$

We will ignore terms that are $O\left(\delta^{2}\right)$ in the equation of motion, and terms that are $O(\delta)$ in Ohm's law.

Using Eq. (4a), the ion velocity is

$$
\mathbf{V}_{i}=\mathbf{V}_{\| i}+\mathbf{V}_{E}+O(\delta) \text {. }
$$

Consistent with Eq. (26), we ignore the terms in the ion velocity that are $O(\delta)$. Then the electron velocity is

$$
\mathbf{V}_{e}=\mathbf{V}_{i}-\frac{1}{n} \mathbf{J}
$$

Thus in MHD the electrons and ions flow as separate fluids, with the differences in their parallel and perpendicular velocities contained in the current density $\mathbf{J}$. The generalized Ohm's law is then

$$
\mathbf{E}=-\mathbf{V}_{i} \times \mathbf{B}+\frac{1}{n} \underbrace{\mathbf{J} \times \mathbf{B}}_{O(\delta)}-\delta^{\frac{1}{n}} \nabla p_{e}=-\mathbf{V}_{i} \times \mathbf{B}+O(\delta) .
$$

However, from Eqs. (27e) and (28a), the second term on the right hand side is $O(\delta)$ and can be ignored. Thus the MHD Ohm's law becomes

$$
\mathbf{E}=-\mathbf{V}_{i} \times \mathbf{B} .
$$

The lack of Whistler and diamagnetic terms in the MHD model comes about from the force balance requirement $\mathbf{J}$ $\times \mathbf{B} \sim O(\delta)$, and not because the ions and electrons flow as a "single" fluid.

In the banana regime, Eq. (28a) is

$$
\mathbf{J} \times \mathbf{B}=\delta\left(n \frac{d \mathbf{V}_{i}}{d t}+\nabla p+\nabla \cdot \Pi_{i}^{\mathrm{nc}}\right),
$$

so that the neoclassical, but not the gyro-viscous, stress appears.

\section{E. The drift ordering}

A consequence of the MHD ordering, Eq. (26), is that force imbalance (in this case, deviation from a force-free state) can occur at first order in $\delta$. In the drift ordering, we envision a situation in which the equilibrium pressure and Lorentz forces are of the same order and remain in almost perfect balance for all times. Motions away from this state will be slow and exceedingly low frequency. This leads to the choice

$$
\varepsilon \sim \delta^{2}, \quad \xi \sim \delta
$$

Then the form of the continuity equation remains unchanged from the MHD model, Eq. (27a), while the expressions of total and electron momentum in the banana regime become

$$
\begin{aligned}
-\nabla p+\mathbf{J} \times \mathbf{B}= & \delta^{2}\left(n \frac{d \mathbf{V}_{i}}{d t}+\nabla \cdot \Pi_{i}^{\mathrm{gv}}+\nabla \cdot \Pi_{i}^{\mathrm{nc}}\right. \\
& \left.-n \mu_{A} \nabla^{2} \mathbf{V}_{i}\right)+O\left(\delta^{4}\right)
\end{aligned}
$$

and

$$
\mathbf{E}=-\mathbf{V}_{e} \times \mathbf{B}-\frac{1}{n} \nabla p_{e}-\nabla \cdot \Pi_{e}^{\mathrm{nc}}
$$

Here we have ordered the artificial viscosity as $\mu_{A} \sim \delta^{2}$ so that it appears at the same order as the acceleration in Eq. (34a). As anticipated, the pressure and Lorentz forces are now comparable and deviations from force balance occur at $O\left(\delta^{2}\right)$. Note that the neoclassical ion parallel viscous force enters at the same order as the gyro-viscous stress. The last term in Eq. (34b) is responsible for the bootstrap current.

Ampere's law is now

$$
\mathbf{J}=\delta \nabla \times \mathbf{B} .
$$

The ion velocity in the drift ordering is

$$
\mathbf{V}_{i}=\mathbf{V}_{E}+\mathbf{V}_{*_{i}}+\mathbf{V}_{\pi i}+\mathbf{V}_{\| i}+O\left(\delta^{2}\right),
$$

where

$$
\mathbf{V}_{*_{i}}=\frac{1}{n B^{2}} \mathbf{B} \times \nabla p_{i}
$$

and 


$$
\mathbf{V}_{\pi i}=\frac{1}{n B^{2}} \mathbf{B} \times \nabla \cdot \Pi_{i}^{\mathrm{gv}}
$$

are the ion diamagnetic and "stress" drift velocities, respectively. The electron velocity is

$$
\mathbf{V}_{e}=\mathbf{V}_{i}-\frac{1}{n} \mathbf{J}
$$

The appearance of the gyro-viscous stress in the ion momentum equation is a consequence of the FLR corrections. Note that the gyro-viscous force appears at the same order as the advective acceleration. In the drift ordering, it is inconsistent to retain $\mathbf{V} \cdot \nabla \mathbf{V}$ while ignoring $\nabla \cdot \Pi_{\mathrm{gv}}$.

\section{F. The standard drift model}

While the drift ordering introduced in Sec. II E produced the lowest order FLR corrections to the fluid equations, it resulted in more complicated equations than in the MHD ordering. In contrast to the drift ordering, the drift model makes use of the velocity decomposition given by Eqs. (36a)-(36d), along with a remarkable result called the gyroviscous cancellation, to produce a simplified set of equations. tion

The standard drift model makes the velocity transforma-

$$
\begin{aligned}
& \mathbf{V}_{i}=\mathbf{V}_{\| i}+\mathbf{V}_{E}+\mathbf{V}_{*_{i}}+O\left(\delta^{2}\right) \\
& \mathbf{V}_{e}=\mathbf{V}_{i}-\frac{1}{n} \mathbf{J}=\mathbf{V}_{\| i}+\mathbf{V}_{E}+\mathbf{V}_{*_{i}}-\frac{1}{n} \mathbf{J}+O\left(\delta^{2}\right)
\end{aligned}
$$

The motivation is to arrive at a set of equations that look like the MHD equations with corrections. Using this last result in the electron equation, Eq. (34b), we have

$$
\begin{aligned}
& \mathbf{E}=-\left(\mathbf{V}_{E}+\mathbf{V}_{*_{i}}-\frac{1}{n} \mathbf{J}_{\perp}\right) \times \mathbf{B}-\frac{1}{n} \nabla p_{e}+O\left(\delta^{2}\right), \\
& =-\mathbf{V}_{E} \times \mathbf{B}-\frac{1}{n} \nabla_{\|} p_{e}+\frac{1}{n}(\underbrace{-\nabla_{\perp} p+\mathbf{J} \times \mathbf{B}}_{O\left(\delta^{2}\right)})+O\left(\delta^{2}\right), \\
& =-\mathbf{V}_{E} \times \mathbf{B}-\frac{1}{n} \nabla_{\|} p_{e} .
\end{aligned}
$$

This is the generalized Ohm's law in the drift model. Notice that the high frequency Whistler response, which comes from $\mathbf{J} \times \mathbf{B}$, has been dropped as a higher order $\left[O\left(\delta^{2}\right)\right]$ correction due to force balance at this order. (A similar argument led to the elimination of Whistler waves from the MHD model.) This is consistent with the low frequency ordering $\omega \sim \delta^{2} \Omega$; the Whistler branch merges with the Alfvén branch at low frequency. The ion equation becomes

$$
\begin{aligned}
\delta^{2}[n & \left.\frac{d}{d t}\left(\mathbf{V}_{\| i}+\mathbf{V}_{E}\right)+n \frac{d \mathbf{V}_{*_{i}}}{d t}+\nabla \cdot \Pi_{i}^{\mathrm{gv}}\left(\mathbf{V}_{i}\right)\right]=-\nabla p+\mathbf{J} \\
& \times \mathbf{B}-\delta^{2} \nabla \cdot \Pi_{i}^{\mathrm{nc}}+\delta^{2} n \mu_{A} \nabla^{2} \mathbf{V}_{i}+O\left(\delta^{4}\right),
\end{aligned}
$$

where we have retained the notation $d / d t=\partial / \partial t+\mathbf{V}_{i} \cdot \nabla$.
One reason for the utility of the drift model is an enormous simplification of the equation of motion that occurs because, in the proper reference frame, the parallel ion gyroviscous force $\nabla \cdot \Pi_{\mathrm{gv}}$ algebraically cancels a significant part of the advective acceleration $n \mathbf{V}_{i} \cdot \nabla \mathbf{V}_{i}$. The remaining terms primarily introduce a slight modification to the total pressure.

The gyro-viscous cancellation may be written as ${ }^{9}$

$$
n\left(\frac{\partial \mathbf{V}_{*_{i}}}{\partial t}+\mathbf{V}_{i} \cdot \nabla \mathbf{V}_{*_{i}}\right)+\nabla \cdot \Pi_{i}^{\mathrm{gv}}\left(\mathbf{V}_{i}\right) \approx \nabla \chi-\mathbf{b} n \mathbf{V}_{*_{i}} \cdot \nabla V_{\| i}
$$

where

$$
\chi=-p_{i} \mathbf{b} \cdot\left(\nabla \times \mathbf{V}_{\perp i}\right)
$$

is a scalar related to the parallel component of the ion vorticity. Both terms involved in the cancellation result from the transport by advection at the streaming velocity $\left(\mathbf{V}_{i}\right)$ of momentum inherent in the gyro-motion $\left(\mathbf{V}_{*_{i}}\right)$. These fluxes almost cancel because the diamagnetic drift does not correspond to any real drift of the guiding centers. Both the gyroviscous force and the advective term $\mathbf{V}_{*_{i}} \cdot \nabla \mathbf{V}_{i}$ explicitly contain the pressure, the velocity, and two gradients, so one can surmise (at least mathematically) how this cancellation might come about. ${ }^{9}$ However, the actual calculation is extremely complex and tedious, ${ }^{6,7,9}$ and seems to have been carried out only under restricted conditions (i.e., uniform magnetic field, sheared slab geometry, uniform temperature, etc.). Further, there is not universal agreement on the exact form of the cancellation. Some authors ${ }^{6,7}$ find additional terms on the right hand side of Eq. (40).

The gyro-viscous cancellation cannot occur in either Hall or ideal MHD, since the advective acceleration and the gyro-viscous force enter at different orders in each of those models.

Using the gyro-viscous cancellation, Eq. (40), in the momentum equation, Eq. (39), and assuming that the magnetic field is approximately constant (so that the unit vector $\mathbf{b}$ can be moved freely through derivative operators), we find the following expressions for the perpendicular and parallel momentum balance:

$$
\begin{aligned}
\left.n \delta^{2} \frac{d \mathbf{V}_{E}}{d t}\right|_{\mathrm{MHD}}= & -\delta^{2} n \mathbf{V}_{*_{i}} \cdot \nabla \mathbf{V}_{E}-\delta^{2} n \mathbf{V}_{\| i} \cdot \nabla \mathbf{V}_{E} \\
& +\delta^{2} n \mu_{A} \nabla^{2} \mathbf{V}_{E}-\delta^{2}(\mathbf{I}-\mathbf{b} \mathbf{b}) \cdot \nabla \cdot \Pi_{i}^{\mathrm{nc}} \\
& -\nabla_{\perp}\left[p\left(1+\delta^{2} \chi\right)\right]+\mathbf{J} \times \mathbf{B}, \\
\left.n \delta^{2} \frac{d \mathbf{V}_{\| i}}{d t}\right|_{\mathrm{MHD}}= & -\delta^{2} n \mathbf{V}_{\| i} \cdot \nabla \mathbf{V}_{\| i}+\delta^{2} n \mu_{A} \nabla^{2} \mathbf{V}_{\| i} \\
& -\delta^{2} \mathbf{b} \cdot \nabla \cdot \Pi_{i}^{\mathrm{nc}}-\mathbf{b} \cdot \nabla\left[p\left(1+\delta^{2} \chi\right)\right],
\end{aligned}
$$

where

$$
\left.\frac{d}{d t}\right|_{\mathrm{MHD}} \equiv \frac{\partial}{\partial t}+\mathbf{V}_{E} \cdot \nabla
$$

These equations differ from the MHD model primarily through a modification to the scalar pressure and the appear- 
ance of a source term proportional to $\mathbf{V}_{*_{i}}$. This last term explicitly introduces the diamagnetic drift frequency $\left(\omega_{*_{i}}\right.$ $\left.\sim \mathbf{V}_{*_{i}} \cdot \nabla\right)$. Note that the dependent perpendicular velocity variable is $\mathbf{V}_{E}$, the $\mathbf{E} \times \mathbf{B}$ drift (or MHD) velocity, and not the actual center of mass velocity.

The drift model thus naturally produces a set of equations that (a) explicitly contain the lowest order FLR corrections to the MHD model, (b) look very much like the MHD equations when cast in terms of the velocity $\mathbf{V}=\mathbf{V}_{E}+\mathbf{V}_{\| i}$, (c) separate easily into perpendicular and parallel parts, and (d) remove most of the complications of the gyro-viscous stress [see Eqs. (32)] and eliminate the Whistler branch. It has proven to be powerful for the analysis of hot, well confined plasmas, such as in a tokamak.

There are, however, some caveats in the drift model. In the first place, the derivation formally admits only very slow flows, which is consistent with the result of force balance through second order in $\delta$. Since this ordering refers to the total ion velocity $\mathbf{V}_{i}$ [see Eqs. (33) and (36a)], it is applicable to both the MHD velocity $\mathbf{V}_{E}$ and the drift velocity $\mathbf{V}_{*}$. Second, the assumption of very low frequency may limit the validity of the model to time scales much longer than the Alfvén transit time $L / V_{A}$. Acceptable frequencies are on the order of the diamagnetic drift frequency $\omega_{*_{i}} \sim V_{*_{i}} / L \ll \omega_{A}$. Third, the form of the gyro-viscous cancellation used here assumes a uniform magnetic field, or a sheared slab. The equations are valid only in the drift ordering, and should be considered only approximate; they are not generally applicable to situations where significant deviations from force balance occur (such as, for example, during the highly nonlinear evolution of instabilities).

We remark that an alternative form of the drift model has been derived ${ }^{12}$ in which a generalized drift velocity is defined that is accurate to all orders in $\delta$. The advantage of this model is that the electron dynamics become exact (within the context of $\left.m_{e}=0\right)$. In principle, the Whistler branch should then reappear in the ion dynamics, but it is again removed by force balance in the drift ordering. It appears at $O\left(\delta^{4}\right)$ and is dropped. The entire model remains accurate to $O\left(\delta^{2}\right)$.

\section{DISPERSIVE MODES AND STABILITY IN EXTENDED MHD}

The drift model provides a useful description for the dynamics of a plasma that never deviates significantly from strict equilibrium. While this is extremely useful for tokamaks, it is too restrictive to provide a generally useful model for a variety of plasma configurations. A widely used model is extended $M H D$, which includes all the terms of the drift ordering, but does not explicitly assume force balance to a certain order in $\delta$, does not employ the gyro-viscous cancellation, and remains in the ion (or momentum carrying) frame of reference. This is

$$
\frac{\partial n}{\partial t}+\nabla \cdot \mathbf{V}=0
$$

$$
\begin{aligned}
& M n \frac{d \mathbf{V}}{d t}=-\nabla p+\mathbf{J} \times \mathbf{B}-\nabla \cdot \Pi_{\mathrm{visc}}-\nabla \cdot \Pi_{\mathrm{gv}}-\nabla \cdot \Pi_{i}^{\mathrm{nc}}, \\
& \mathbf{E}=-\mathbf{V} \times \mathbf{B}+\frac{1}{n e}\left[\mathbf{J} \times \mathbf{B}-\nabla p_{e}-\nabla \cdot \Pi_{e}^{\mathrm{nc}}\right]+\eta \mathbf{J},
\end{aligned}
$$

where the components of the stress tensor are given in Sec. II. [Here, and for the remainder of this section, we revert to dimensional equations. For completeness, we have also included the resistive contribution to the generalized Ohm's law, Eq. (44c), which was omitted from the discussion in Sec. II.] The expressions for the neoclassical stresses are dependent on the peculiarities of the plasma being modeled, and may be omitted when they are inappropriate.

The standard MHD equations admit several normal modes of oscillation. They all have the common property that $\omega \sim k$. An explicit computational approach to the solution of the MHD equations is then subject to the Courant stability condition $\Delta t<\Delta x$. While this can be quite restrictive for studies of low frequency phenomena in the presence of fast waves (as is common in modeling highly magnetized plasmas), it may be tolerated for the study of fast phenomena, such as implosions and shock waves. However, the additional terms of the extended MHD model introduce new dispersive normal modes with the property $\omega \sim k^{2}$. This has significant implications for computational modeling, since the corresponding Courant condition, $\Delta t<\Delta x^{2}$, is too restrictive for explicit methods to be useful for most practical problems. Here we examine the dispersion properties of these waves in more detail. Algorithms for dealing with them will be discussed in Sec. IV.

\section{A. Dispersion from Ohm's law}

To examine the dispersive waves of extended MHD, it is instructive to consider the generalized Ohm's law without assuming force balance to $O\left(\delta^{2}\right)$. From the derivation preceding Eq. (38),

$$
\mathbf{E}=-\mathbf{V} \times \mathbf{B}-\frac{1}{n e} \nabla_{\|} p_{e}+\frac{1}{n e}\left[-\nabla_{\perp} p+\mathbf{J} \times \mathbf{B}\right] .
$$

Recall that the term in brackets on the right hand side of Eq. (45) is ordered out of the drift model [see Eq. (38)]. The first term on the right hand side yields the usual MHD Alfvén waves. The remaining terms are FLR corrections. The second term $\left(\sim \nabla_{\|} p_{e}\right)$ (when combined with other terms) introduces kinetic Alfvén waves (KAW), and the last term $(\sim \mathbf{J}$ $\times$ B) introduces Whistler waves.

In a uniform, homogeneous plasma with straight field lines, the linearized Whistler wave equation comes about from combining Eq. (45) with Faraday's law and Ampere's law,

$$
\frac{\partial^{2} \mathbf{B}}{\partial t^{2}}=\left(\frac{V_{A}^{2}}{\Omega}\right)^{2}(\mathbf{b} \cdot \nabla)^{2} \nabla \times \nabla \times \mathbf{B} .
$$

These waves have the dispersion relation 


$$
\omega^{2}=\left(\frac{V_{A}^{2}}{\Omega}\right)^{2} k^{2} k_{\|}^{2}
$$

so that shorter wavelengths have quadratically higher frequencies. This is the source of the computational problem for explicit algorithms.

Under similar conditions, but assuming overall incompressibility, the KAW arises from a combination of the second term in Eq. (45), Faraday's law, Ampere's law, the perpendicular momentum equation, and the electron energy equation. Under these conditions, the KAW wave equation is

$$
\frac{\partial^{2} \mathbf{B}}{\partial t^{2}}=\left(\frac{V_{A} V_{t h}}{\Omega}\right)^{2}(\mathbf{b} \cdot \nabla)^{2} \nabla \times[\mathbf{b} \mathbf{b} \cdot \nabla \times \mathbf{B}],
$$

where $V_{\mathrm{th}} *$ is the thermal speed evaluated with the electron temperature and the ion mass. The KAW dispersion relation is

$$
\omega^{2}=\left(\frac{V_{A} V_{\mathrm{th}}}{\Omega}\right)^{2} k_{\perp}^{2} k_{\|}^{2} .
$$

Whistler waves come from a combination of the generalized Ohm's law and Faraday's law. They are solely related to the electrons. The KAW involves both electron and ion dynamics, and their frequency is low enough to be captured in the drift ordering.

\section{B. Dispersion from gyro-viscosity}

The gyro-viscous force is not dissipative because it is not caused by particle collisions $\left(\eta_{3}\right.$ is independent of the collision frequency $\nu$ ); it represents a momentum transport inherent in the gyro-motion of the particles, which is reversible. [It can be shown explicitly from Eq. (8b) that the work done by the gyro-viscous force vanishes, i.e., $\nabla \mathbf{v}: \Pi_{\mathrm{gv}}=0$.]

Consider a uniform plasma with straight field lines and no zero-order gradients or current. We choose a Cartesian coordinate system $(x, y, z)$ with the $z$ axis positively aligned with the mean magnetic field. We note that the $-\nabla p_{e} / n$ contribution to Ohm's law is annihilated by the curl operator even in the presence of density fluctuations because of the assumed uniformity of the background electron pressure. Similar comments apply to the $\nabla \cdot(\mathbf{J} / n)$ contribution to the electron pressure. We can therefore omit density evolution from the stability analysis; the perturbed density can be determined a posteriori. This allows use of a single adiabatic energy equation.

The extended MHD equations, including the gyroviscosity, can then be linearized, and the dispersion relations are easily obtained for the special cases of parallel and perpendicular propagation. For the case of parallel propagation, the analysis is simplified by defining new variables $V_{ \pm}$ $=V_{x} \pm i V_{y}$ and $B_{ \pm}=B_{x} \pm i B_{y}$. Then we find

$$
i \omega V_{ \pm}=\frac{i B_{0} k_{z}}{\mu_{0} n e} B_{ \pm} \pm \frac{i \eta_{4} k_{z}^{2}}{n m} V_{ \pm},
$$

$$
i \omega B_{ \pm}=i B_{0} k_{z} V_{ \pm} \pm \frac{i B_{0} k_{z}^{2}}{\mu_{0} n e} B_{ \pm} .
$$

Equations (50a) and (50b) are two sets of coupled equations, one for the pair $\left(V_{+}, B_{+}\right)$, and the other for the pair $\left(V_{-}, B_{-}\right)$. They represent right $(-)$ and left $(+)$ polarized waves. Defining

$$
\begin{aligned}
& \omega_{A}=\frac{B_{0}\left|k_{z}\right|}{\sqrt{\mu_{0} m n}}=V_{A}\left|k_{z}\right|, \\
& \omega_{4}=\frac{\eta_{4} k_{z}^{2}}{m n}=\frac{V_{\mathrm{th}_{i}}^{2}}{2 \Omega} k_{z}^{2}=\frac{1}{2}\left(\rho_{i} k_{z}\right)^{2} \Omega,
\end{aligned}
$$

and

$$
\omega_{W}=\frac{B_{0} k_{z}^{2}}{\mu_{0} n e}=\left(\frac{\omega_{A}}{\Omega}\right)^{2} \Omega=\frac{1}{\beta}\left(\rho_{i} k_{z}\right)^{2} \Omega,
$$

the parallel dispersion relation is obtained as

$$
\left(\omega \pm \omega_{4}\right)\left(\omega \pm \omega_{W}\right)=\omega_{A}^{2},
$$

where the $+(-)$ sign refers to the solution corresponding to $V_{-}$and $B_{-}\left(V_{+}\right.$and $\left.B_{+}\right)$, and $\rho_{i}=V_{t h_{i}} / \Omega$ is the ion Larmor radius. There are thus four independent solutions, two for the left polarized wave, and two for the right.

Assuming $\rho_{i} k_{z} \ll 1$, the solutions for the left and right polarized waves can be written as

$$
\left.\left.\omega_{L \pm}=V_{A} k_{z}\right\rfloor \pm 1+\frac{1+\beta}{2 \sqrt{\beta}}\left(\rho_{i} k_{z}\right)\right\rfloor
$$

and

$$
\omega_{R \pm}=V_{A} k_{z}\left\lfloor \pm 1-\frac{1+\beta}{2 \sqrt{\beta}}\left(\rho_{i} k_{z}\right)\right\rfloor .
$$

The Whistler result is obtained by setting $1+\beta \rightarrow 1$ in the numerator of the last terms. The gyro-viscosity thus gives an $O(\beta)$ correction to the parallel waves.

For the case of perpendicular propagation, the result is

$$
\frac{\omega^{2}}{\omega_{s}^{2}+\omega_{A}^{2}}=1+\frac{\omega_{3}^{2}}{\omega_{s}^{2}+\omega_{A}^{2}},
$$

where $\omega_{s}^{2}=C_{s}^{2} k_{x}^{2}, C_{s}$ is the sound speed, and $\omega_{3}=\omega_{4} / 2$. This is a modification of the fast magnetosonic wave. The frequency can be expressed as

$$
\omega^{2}=V_{A}^{2} k_{x}^{2}\left\lfloor 1+\frac{\gamma \beta}{2}+\frac{\beta}{16}\left(\rho_{i} k_{x}\right)^{2}\right\rfloor,
$$

so that the mode is now dispersive.

These dispersive waves may appear as the lowest order FLR correction in the warm plasma dispersion relation derived strictly from kinetic theory. ${ }^{38}$

\section{Stabilizing effects of extended MHD}

The extended MHD model admits high frequency dispersive waves that present challenges for long time scale computational modeling. However, the significance of the extended MHD model is not in these stable waves, but in the 
stabilizing effect these modes can have on MHD instabilities. These effects are important to capture in any computational model of fusion plasmas. Here we illustrate the physics of these effects by considering one of the simplest MHD instabilities: the support of a density gradient in a gravitational field in the presence of a magnetic field, as first studied by Roberts and Taylor. ${ }^{30}$ This serves as a paradigm for FLR and two-fluid stabilization in more complex situations.

We consider the problem of a heavy fluid supported by a light fluid in the presence of a gravitational force. The problem is two-dimensional in the $(x, y)$ plane, with the gravitational acceleration $\mathbf{g}$ pointing in the negative $x$ direction, and the density gradient pointing in the positive $x$ direction. We also assume an exponentially increasing density profile. Thus $\mathbf{G}=\rho \mathbf{g}=-\rho g \mathbf{e}_{x}$ and $\nabla \rho=\eta \rho \mathbf{e}_{x}$, where $\eta=1 / L_{n}$ and $L_{n}$ is the equilibrium density scale length. The equilibrium magnetic field is in the $z$ direction, and any velocities are in the $(x, y)$ plane. Including two-fluid and FLR effects, the continuity and momentum equations, and Ohm's law, are then

$$
\begin{aligned}
& \frac{\partial \rho}{\partial t}+\nabla \cdot \rho \mathbf{V}=0, \\
& \rho \frac{d \mathbf{V}}{d t}=-\nabla\left(p+\frac{B^{2}}{2 \mu_{0}}\right)+\rho \mathbf{g}-\nabla \cdot \Pi,
\end{aligned}
$$

and

$$
\mathbf{E}=-\mathbf{V} \times \mathbf{B}-\frac{M}{\rho e}\left|\nabla\left(\frac{B^{2}}{2 \mu_{0}}\right)+\nabla p_{e}\right|,
$$

where $p$ is the total fluid pressure, $p_{e}$ is the electron pressure, $M$ is the ion mass, and $\Pi$ is the gyro-viscous stress tensor, given by Eq. (8b).

In order for the square of the sound speed, $C_{s}^{2}$ $=(\partial p / \partial \rho)_{S}$, to be positive, we require that the equilibrium pressure be an increasing function of the $x$ coordinate. If we assume an equation of state of the form $p=p(\rho)$ (a barotropic fluid, which encompasses isothermal and adiabatic fluids as special cases), then the magnetic field variation required for equilibrium force balance depends on the specific form of $p(\rho)$.

Using Eq. (60), Eq. (61) can be written as

$$
\left.\mathbf{E}=-\mathbf{V} \times \mathbf{B}+\frac{M}{\rho e} \mid \rho \frac{d \mathbf{V}}{d t}+\nabla p_{i}-\rho \mathbf{g}+\nabla \cdot \Pi\right\rfloor,
$$

where $p_{i}$ is the ion pressure, so that the magnetic field only explicitly enters the dynamics through the total pressure $p_{T}$ $=p+B^{2} / 2 \mu_{0}$. Therefore, as far as the dynamics are concerned, perturbations to the magnetic field can be ignored, and all perturbed pressure forces can be viewed as entering through the fluid pressure $p$. (Following Roberts and Taylor, ${ }^{30}$ we also assume that the equilibrium magnetic field is "approximately constant.") It is then a significant, and consistent, simplification to assume that all perturbations are electrostatic, so that $\nabla \times \mathbf{E}=0$. If we further assume that the fluid is isothermal, then $C_{s}^{2}=$ const. Setting the curl of Eq. (62) to zero, and assuming that the ions are barotropic, yields

$$
\nabla \cdot \mathbf{V}+\frac{1}{\Omega} \nabla \times \frac{d \mathbf{V}}{d t}-\frac{1}{\Omega \rho^{2}} \nabla \rho \times \nabla \cdot \Pi=0 .
$$

(Of course, there would be additional terms if there were variation along the field, or if the field were not constant.) Equations (59), (60), and (63) are four equations in the four unknowns $\rho, \mathbf{V}$ (two components), and $p_{T}=p+B^{2} / 2 \mu_{0}$. Equation (63) (or, equivalently, $\nabla \times \mathbf{E}=0$ ) serves as an "equation of state" to close the system and determine $p_{T}$. This is analogous to the assumption of incompressibility in hydrodynamics, except that the fluid is no longer strictly incompressible.

We linearize about the equilibrium state, assuming variations of the form $\exp (i \omega t+i k y)$. We ignore explicit variations of the coefficients in the $x$ direction, which requires $\eta L_{x}$ $\ll 1$, where $L_{x}$ is the maximum value of $x$. With $L_{x} \sim \lambda_{y}$ $=2 \pi / k$, this implies $\eta \ll|k|$. The linearized components of the gyro-viscous stress are

$$
(\nabla \cdot \Pi)_{x}=-\left(\rho_{0} \nu_{0}\right)^{\prime} i k V_{x}+\rho_{0} \nu_{0} k^{2} V_{y}
$$

and

$$
(\nabla \cdot \Pi)_{y}=-\left(\rho_{0} \nu_{0}\right)^{\prime} i k V_{y}-\rho_{0} \nu_{0} k^{2} V_{x},
$$

where (..)' indicates differentiation with respect to $x$, and $\nu_{0}=\Omega_{0} \rho_{i}^{2} / 2$. Now, we let the entire variation of the gyroviscous coefficient enter through the equilibrium density $\rho_{0}$, so that $\nu_{0}=$ const. (This implies constant temperature and ignores the variation of $\Omega_{0}$ with $x$, but is consistent with the assumption that $B_{0}$ is essentially uniform.) With this, the final set of linearized equations is

$$
\begin{aligned}
& i \omega \rho+\eta \rho_{0} V_{x}+i k \rho_{0} V_{y}=0, \\
& \frac{g}{\rho_{0}} \rho+\left(i \omega-\zeta \eta \nu_{0} i k\right) V_{x}+\zeta \nu_{0} k^{2} V_{y}=0, \\
& -\zeta \nu_{0} k^{2} V_{x}+\left(i \omega-\zeta \eta \nu_{0} i k\right) V_{y}+\frac{i k}{\rho_{0}} p_{T}=0, \\
& \left(\xi \frac{\omega k}{\Omega_{0}}+\xi \zeta \frac{\nu_{0} \eta}{\Omega_{0}} k^{2}\right) V_{x}+\left(1+\xi \zeta \frac{\eta^{2} \nu_{0}}{\Omega_{0}}\right) i k V_{y}=0 .
\end{aligned}
$$

We have introduced the parameters $\xi$ and $\zeta$ so that $\xi=0, \zeta$ $=0$ indicates ideal MHD, $\xi=1, \zeta=0$ indicates extended Ohm's law but no gyro-viscosity, $\xi=0, \zeta=1$ indicates gyroviscosity but no extended Ohm's law, and $\xi=1, \zeta=1$ indicates both extended Ohm's law (two-fluid) and gyro-viscous (FLR) effects, i.e., extended MHD.

Solutions of Eqs. (66)-(69) are summarized in Table I. The MHD result shows unconditional instability under these conditions. Both two-fluid and gyro-viscous (FLR) effects are stabilizing for sufficiently large wave number. This is an example of extended MHD effects altering low frequency dynamics, and is the reason parasitic waves must be retained in the formulation.

The primary difference between the ideal MHD and twofluid models in this geometry is the role of compressibility. In MHD, the electrostatic assumption and the form of Ohm's law require $\nabla \cdot \mathbf{V}=0$. A nonsolenoidal velocity field would 
TABLE I. Extended MHD stabilization of the gravitational interchange instability

\begin{tabular}{|c|c|c|c|}
\hline Model & Dispersion relation & Solution & $\begin{array}{l}\text { Stabilizing wave } \\
\text { number }\end{array}$ \\
\hline $\begin{array}{c}\text { MHD } \\
(\xi=0, \zeta=0)\end{array}$ & $\omega^{2}+g \eta=0$ & $\omega=i \sqrt{g \eta}$ & None \\
\hline $\begin{array}{l}\text { Two-fluid } \\
(\xi=0, \zeta=1)\end{array}$ & $\omega^{2}-\frac{g k}{\Omega_{0}} \omega+g \eta=0$ & $\begin{array}{c}2 \omega=\frac{g k}{\Omega_{0}} \\
\pm \sqrt{\left(\frac{g k}{\Omega_{0}}\right)^{2}-4 g \eta}\end{array}$ & $k^{2}>\frac{4 \eta \Omega_{0}^{2}}{g}$ \\
\hline $\begin{array}{c}\text { Gyro-viscosity } \\
(\xi=1, \zeta=0)\end{array}$ & $\omega^{2}-\nu_{0} \eta k \omega+g \eta=0$ & $\pm \sqrt{\left(\omega=\nu_{0} \eta k\right.}$ & $k^{2}>\frac{4 g}{\nu_{0}^{2} \eta}$ \\
\hline $\begin{array}{l}\text { Full extended } \\
\qquad \begin{array}{l}\text { MHD } \\
(\xi=1, \zeta=1)\end{array}\end{array}$ & $\omega^{2}-\left(\frac{g k}{\Omega_{0}}+\nu_{0} \eta k\right) \omega+g \eta=0$ & $\begin{array}{c}2 \omega=\frac{g k}{\Omega_{0}}+\nu_{0} \eta k \\
\pm \sqrt{\left(\frac{g k}{\Omega_{0}}+\nu_{0} \eta k\right)^{2}-4 g \eta}\end{array}$ & $k^{2}>\frac{4 g \eta}{\left(\frac{g}{\Omega_{0}}+\nu_{0} \eta\right)}$ \\
\hline
\end{tabular}

result in compression of the magnetic field. As a consequence, the electron and ion motions are tied together, moving with their common $\mathbf{E} \times \mathbf{B}$ drift in the $x$ direction. The two-fluid treatment allows the decoupling of the electron and ion motions because of their separate gravitational drifts. Consider an element of the ion fluid moving into a region of lower (or higher) electron density. If the ion fluid were incompressible, this would result in charge imbalance. The ion fluid element must therefore be compressed (or expanded) just enough to maintain charge neutrality. Nonsolenoidal ion flow is thus a requirement of quasineutrality; it is enabled by the perturbed ion drifts, as given by Eq. (63). The $y$ component of the perturbed ion flow does work on the perturbed electric field. Since $E_{y}$ increases with $k$, this implies a wave number at which there is insufficient free energy to drive the instability.

The gyro-viscosity leads to an additional ion drift that can also interact with the perturbed electric field, and modify the stability threshold [see Eqs. (36c) and (63)].

\section{The effect of the ion heat stress tensor}

In Sec. II we recognized that the classical Braginskii expression for the gyro-viscosity is incomplete, and formally should contain additional terms proportional to the ion heat flux. ${ }^{35-37}$ Here we examine the effect of these terms on the propagation of sound waves and the gravitational interchange instability, and show that they are negligible, at least for relatively short wavelengths, in the sense that they do not introduce any new destabilizing or stabilizing influences.

The minimal set of relevant equations to describe sound waves is

$$
\rho_{0} \frac{\partial \mathbf{V}}{\partial t}=-\nabla p-\nabla \cdot \Pi_{\wedge_{q}},
$$

$$
\frac{\partial p}{\partial t}=-\gamma p_{0} \nabla \cdot \mathbf{V}
$$

where $\Pi_{\wedge q}$ is given by Eqs. (17) and (18), and the heat flow is taken to be

$$
\mathbf{q}=-\kappa_{\|} \nabla_{\|} T-\kappa_{\perp} \nabla_{\perp} T-\kappa_{\wedge} \mathbf{b} \times \nabla_{\perp} T .
$$

For a uniform plasma with a constant magnetic field in the $z$ direction, and assuming $\exp \left(i \omega t+i k_{\perp} y+i k_{\|} z\right)$ dependence, the dispersion relation is

$$
\omega^{2}=C_{s}^{2} k^{2}\left[1+\frac{\kappa_{\wedge}}{5 n_{0} \Omega} f(\theta) k^{2}\right],
$$

where $C_{s}^{2}=\gamma p_{0} / \rho_{0}$ is the sound speed, and $\theta$ is the angle between $\mathbf{k}$ and $\mathbf{B}$. The angular dependence is given by

$$
f(\theta)=\frac{1}{2} \sin \theta\left(2 \sin ^{2} \theta+\cos ^{2} \theta-\sin \theta \cos \theta\right),
$$

which has the property that $f(0)=0$ and $f(\pi / 2)=1$.

Equation (73) exhibits a dispersive modification to the sound wave for oblique to normal propagation $(0<\theta$ $\leqslant \pi / 2$ ). However, the propagation of the parallel sound wave $(\theta=0)$ is unaffected (i.e., $\left.\omega^{2} \sim k^{2}\right)$. Note that the dispersive modification depends only on the "cross" component of the ion thermal conductivity. Using the expression $\kappa_{\wedge}$ $=5 n_{0} T / 2 m_{i} \Omega$, Eq. (73) can be written as

$$
\omega^{2}=C_{s}^{2} k^{2}\left[1+f(\theta)\left(\rho_{i} k\right)^{2}\right],
$$

which exhibits the FLR nature of the correction explicitly.

We remark that the expressions for the heat flow and coefficients of thermal conductivity appearing above refer to the ions. Only the ion "cross" thermal conductivity need be considered. Since this is smaller than the electron thermal 
conductivity by a factor of the mass ratio, this effect can be neglected. In particular, more sophisticated nonlocal closure expressions for the parallel electron heat flow will not affect the propagation of sound waves.

With regard to the gravitational interchange instability, including the ion heat stress in the gyro-viscous force, and ignoring the collisional heat flux, yields a dispersion relation that differs from the third entry in Table I by terms that are second order in the small quantities $k a$ and $\eta a$. The heat stress therefore makes a negligible contribution to the gravitational interchange instability, at least for short wavelength.

While the ion heat stress introduces an additional dispersive wave (the modification of the oblique sound wave), it does not have any obvious effect on the stability of low frequency dynamics, and it may be tentatively omitted from the model. However, this conclusion may need to be revisited if effects on long-wavelength stability are subsequently demonstrated.

\section{COMPUTATIONAL MODELS FOR EXTENDED MHD}

\section{A. General considerations}

When developing discrete models for resistive MHD, one is immediately confronted with three properties that make the task extremely difficult.

The first of these is the extreme temporal stiffness exhibited by the normal modes of the MHD equations. One measure of this property is given by the Lundquist number $S$ $=\tau_{R} / \tau_{A}$, the ratio of the time required for resistive diffusion to significantly modify the system to the time required for an Alfvén wave to transit the system. Modern experiments have $S>10^{7}$, often as large as $10^{10}$ or more. Theory ${ }^{39}$ suggests that important physical phenomena grow linearly on a time scale $\tau_{T} / \tau_{A} \sim S^{\alpha}(0<\alpha<1)$, and evolve nonlinearly on a time scale $\tau_{N} / \tau_{A} \sim 0.1 S$. Since versatile computational models must include all the fast time scales, it is necessary to track simultaneously phenomena whose characteristic time scales differ by $5-10$ orders of magnitude. These calculations require special time differencing algorithms, and are extremely challenging.

The second computational difficulty comes about because of the vast differences in spatial scale lengths exhibited by the solutions of the relevant mathematical model. In their linear stage, the spatial structures exhibited by the important physical phenomena are aligned along a magnetic field, and their transverse extent scales as $\varepsilon k L \sim S^{1 / 2}$ (with $\varepsilon$ the layer width, $k$ the parallel wave number, and $L$ a characteristic global scale length), becoming smaller as $S$ increases. Generally, the computational models must resolve both this length scale and the global geometry.

The third computational difficulty arises because of the anisotropy introduced by the presence of a strong magnetic field. As remarked in the previous paragraph, the dynamics relevant to many plasma physics problems tend to be strongly aligned with the magnetic field direction, and quite localized across it. Further, the dispersion relation associated with wave propagation in a magnetized plasma exhibits very distinct polarizations (shear Alfvén, compressional Alfvén, and sound-wave branches in the low-frequency limit). Ana- lytically, these normal modes of oscillation are completely decoupled. An accurate description of these waves is required for a realistic description of the dynamics; many of the important physical phenomena in laboratory plasmas are associated with destabilization of the shear Alfvén branch. Unless care is taken, numerical approximations can cause unphysical coupling between the branches that lead to inaccuracies or even numerical instabilities. (Specifically, the differential operator $\mathbf{B} \cdot \nabla$ is important and must be carefully modeled.) Further, thermal conductivity in magnetized plasmas is often highly anisotropic, being much larger along the field than across it. Even small errors in resolving this anisotropy can produce large, unphysical perpendicular thermal transport that may strongly affect the results of the computation.

For nonideal single fluid MHD computations, these properties dictate the application of specialized methods of spatial and temporal approximation. Finite elements and flux-aligned grids have recently been shown to be valuable for resolving the anisotropic operators (various forms of B $\nabla)$ of nonideal MHD. ${ }^{28}$ Specialized implicit methods have been developed to deal with temporal stiffness, ${ }^{21-28,40-44}$ and have enabled accurate computations to proceed with CFL numbers in excess of $10 .^{4,5}$ While all of these approaches require solution of large algebraic systems at every time step, the Hermitian character of the relevant operators can be used to facilitate computation. ${ }^{45}$ This is particularly important for massively parallel computer architectures, where global communication is computationally costly. The overall effectiveness of implicit methods is demonstrated in Fig. 1, which illustrates the evolution of a resistive tearing mode for very long times using the resistive MHD model. While the early stages of growth are exponential, there is a transition to the slower algebraic growth that characterizes these modes in the early nonlinear stage. ${ }^{46}$ The evolution of the saturated magnetic island has been carried out for a time exceeding the characteristic energy confinement time for the plasma. This demonstrates that, using these strongly implicit methods, dynamical calculations of plasma dynamics on the transport time scale are possible. These methods have been described in detail elsewhere. ${ }^{28}$

In extended MHD, the numerical challenges are compounded by the presence of the new dispersive waves discussed in Sec. III, coupled with the fact that the linear operators for these modes are not self-adjoint. This has required that new approaches for the discrete approximation of the fluid equations be developed and tested. It has also required the implementation of linear solver software for nonHermitian algebraic systems, ${ }^{45,47}$ which are computationally more intensive than those for Hermitian systems. Some of these issues as related to extended MHD are described in the following sections.

\section{B. Spatial discretization}

All of the standard classes of spatial approximationfinite difference, finite volume, finite element, and spectralhave been applied to nonlinear MHD simulation. Spectral methods have traditionally been preferred for periodic coor- 

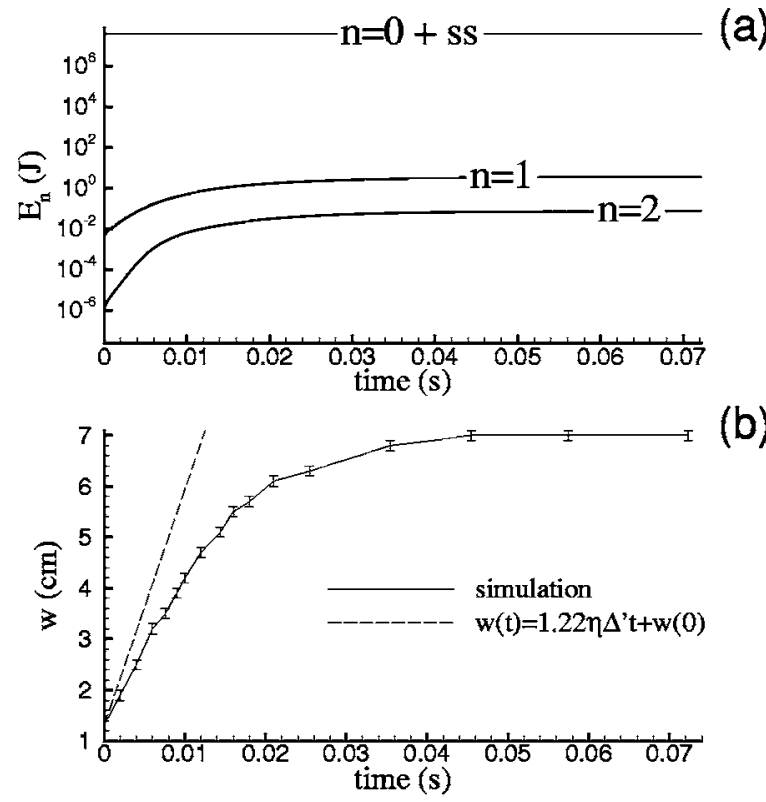

(b)

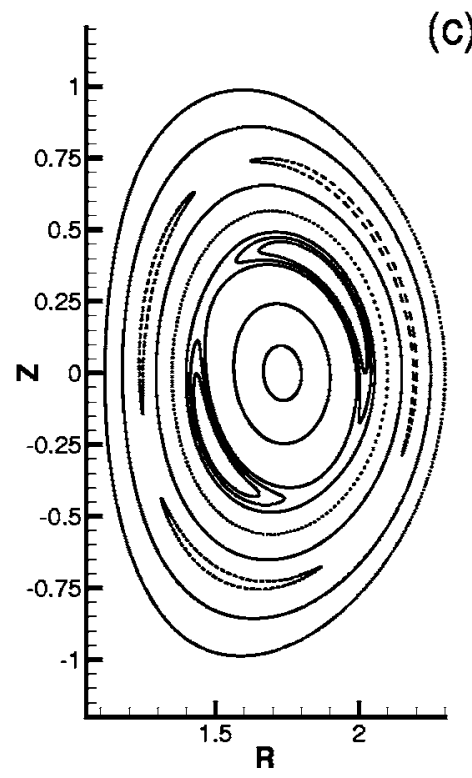

FIG. 1. Simulated nonlinear evolution of a tearing mode in toroidal geometry from Ref. 30. Magnetic energy for each toroidal Fourier component is shown in (a), where $\mathrm{E}_{\mathrm{n}}=\int\left(\mathbf{B}_{s s}+\mathbf{b}_{\mathrm{n}}\right)^{2} / 2 \mu_{0}$ for $\mathrm{n}=0$ and $\mathrm{E}_{\mathrm{n}}=\int \mathbf{b}_{\mathrm{n}}^{*} \cdot \mathbf{b}_{\mathrm{n}} / \mu_{0}$ for $\mathrm{n}=1,2$. The island-width evolution is shown in (b) for the magnetic island at the $q=2$ surface with bars indicating the estimated error in measuring the separatrix from Poincaré surfaces of section. The analytic small-island evolution from Ref. 43 with an estimated $\Delta^{\prime}$ is also plotted in (b). The Poincaré surface of section for the final magnetic configuration is shown in (c).

dinates; however, their primary practical benefit of making simple algebraic operations out of differential operators may be lost when elements of the metric tensor are nonuniform in a periodic coordinate. Thus, while spectral representation of the poloidal angle works well for cylindrical modelling (Ref. 22 , for example), it is less efficient for tori of circular cross section (Refs. 20 and 41 are examples), and only marginally better than other methods for poloidal cross sections with moderate shaping (Refs. 44 and 48 are examples). Similarly, while magnetic confinement configurations are typically toroidal in shape to avoid end losses associated with open systems, spectral methods for the toroidal coordinate work best for toroidally symmetric geometries such as those of tokamaks, reversed-field pinches, and several other devices. Here, it has proven effective to use finite elements for irregular poloidal cross sections with finite Fourier series for the toroidal coordinate. ${ }^{28,49}$ However, for toroidally asymmetric configurations, such as modern stellarators, fully meshed representations are effective. ${ }^{50}$

The highest spatial derivatives in the PDE system for the primitive physical fields [plasma flow velocity $(\mathbf{V})$, magnetic field $(\mathbf{B})$, particle number density $(n)$, and species temperatures $\left(T_{\mathrm{i}}\right.$ and $T_{\mathrm{e}}$ for ions and electrons)] result from collisional effects and are second-order. While the system has a hyperbolic character associated with the propagation of waves, resolving the effects of the second-order terms is usually necessary for the macroscopic dynamics that affect the magnetic configuration. A conforming representation then requires a polynomial basis where first-derivatives are square-integrable. ${ }^{51}$ (Formulation in terms of scalar potential fields can be accomplished using a basis with continuous derivates. ${ }^{52}$ ) For the MHD model, an effective semi-implicit algorithm applies self-adjoint differential operators at the advanced time level, so its weak form constitutes a sequence of variational problems. Adding drift effects breaks this mathematical symmetry, and the weak form is not variational.

As described in Ref. 28, high-order finite-element representations have proven to be useful for extended MHD modeling. First, divergence errors in the computed magnetic field can be made small-a finding that is consistent with analysis (see Ref. 53 and references therein) performed for incompressible fluid computations. Second, it is possible to resolve the extremely anisotropic properties of magnetized plasmas without significant alignment of the magnetic field and the mesh. Third, nonuniform meshing does not upset high-order convergence properties, and the boundary condition treatment does not change with the degree of the polynomial basis functions. Fourth, using isoparametric mappings from element coordinates to physical space ${ }^{51}$ provides an efficient means for representing the curved geometry of the equilibrium state. The results from a resistive MHD benchmark illustrate the effectiveness of the high-order representation with a packed mesh of curved finite elements. The anisotropic nature of the Lorentz force and the resonance condition for the wave-number vector of the helical perturbation and the equilibrium magnetic field, $\mathbf{k} \cdot \mathbf{B}_{0}=0$, lead to the localization shown in Fig. 2, and convergence of the magneticfield solution with different basis functions is shown in Fig. 3 . Tests demonstrating the effectiveness on extremely anisotropic thermal conduction are detailed in Ref. 28.

The spatial representation also affects the manner in which kinetic effects of the electrons and majority ions are incorporated into the modeling. Effects associated with charged particles rapidly streaming along magnetic-field lines are computed through solutions of a drift kinetic equation using the method of characteristics (see Sec. V A) and a basis function expansion in velocity $\operatorname{space}^{54}$ (see Sec. V B). The finite element/finite Fourier series spatial representation provides a meaningful prescription of the fields that influence the kinetic distortions along the characteristic trajectories. 


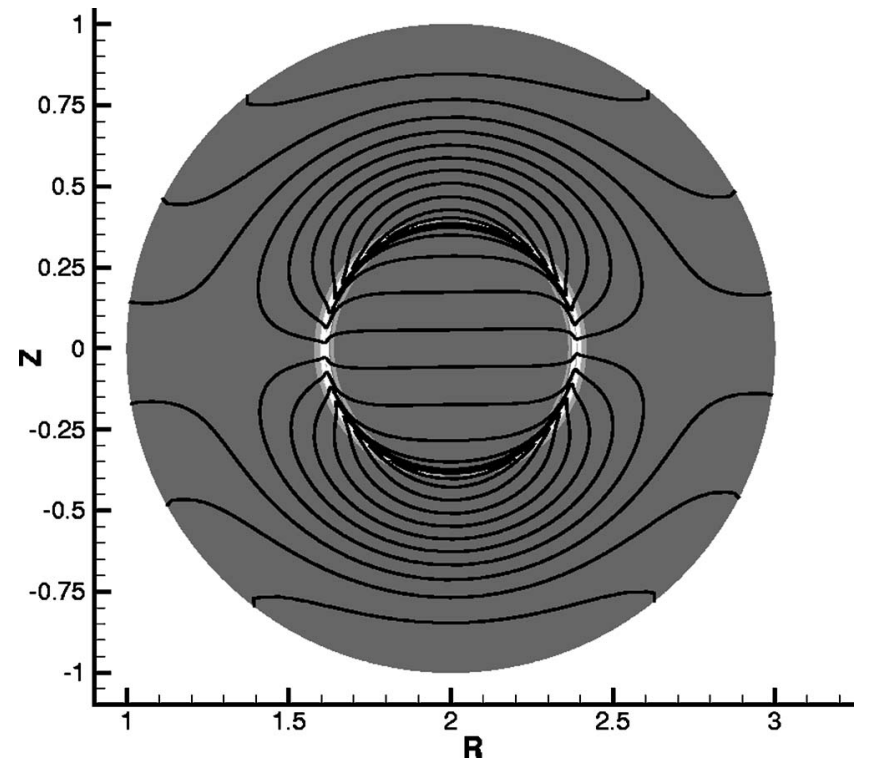

FIG. 2. Cylindrical tearing-mode benchmark results illustrating localization of out-of-plane flow amplitude (lighting intensity) and out-of-plane magnetic field (contour lines) for a helical MHD tearing mode. The mesh is $24 \times 24$ (radial-azimuthal) curved biquartic elements, packed in the radial direction.

\section{Temporal discretization}

An important physical consideration for the time advance is that resistive and viscous dissipation are small in high-temperature plasmas, but they must be treated accurately to reproduce the macroscopic behavior that changes magnetic topology. Fortunately, the dynamics of interest does not include shock propagation, and methods that introduce numerical dispersion to stabilize wave propagation at large time step are suitable. Thus, while purely explicit methods are ruled out by the multiscale nature of the problem, various implicit methods have been applied. A 'quasiimplicit' approach solves the compressive flows that lead to

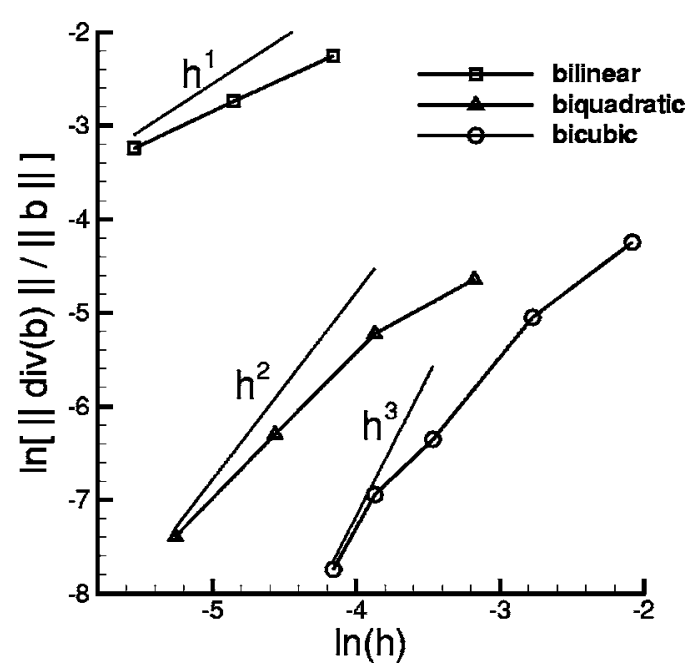

FIG. 3. Convergence studies on the magnetic divergence constraint for a cylindrical tearing-mode benchmark with bilinear, biquadratic, and bicubic finite elements (from Ref. 30). The parameter $h$ is the inverse of the number of elements in each direction, and the convergence rates expected from analysis (for first derivatives of the solution) are illustrated. the fastest waves implicitly and advances other dynamics explicitly. ${ }^{41}$ Another method implicitly advances all dynamics that are linear with respect to an initial equilibrium and explicitly advances all nonlinear dynamics. ${ }^{25}$ The 'semiimplicit' method $^{21-23,28,40,43,55}$ is based on the leapfrog method for wave propagation and in time-discrete form applies a positive-definite self-adjoint differential operator to changes in the flow. The semi-implicit operator can be used to stabilize linear and nonlinear wave propagation, and its spectrum governs temporal convergence properties in multiscale computations. ${ }^{22}$ Time-centered implicit methods are also applicable to nonideal and extended MHD, ${ }^{44,56,57}$ and their use in this area is a subject of active research. The optimal degree of implicitness depends on the efficiency of the various algorithms in performing physically relevant computations and likely depends on both the computer architecture and problem-specific issues.

For the finite-element spatial representation, we focus on an adaptation of the semi-implicit method that has been used successfully with finite-volume spatial representations. ${ }^{22,24} \mathrm{~A}$ benefit that arises with finite elements is that the anisotropic operator that distinguishes the fast, shear, and slow modes of the MHD spectrum ${ }^{43}$ is straightforward to implement and, by construction, results in a Hermitian matrix for the velocity advance. ${ }^{28}$ For the subdominant plasma flow in resistive MHD, predictor-corrector steps for advective terms can be retained. ${ }^{55}$ However, extended-MHD drift effects change the mathematical character of the PDE system, as we have already discussed. We have not found it practicable to retain only self-adjoint operators for large- $\Delta t$ stabilization in semiimplicit extended MHD (contrary to Ref. 58). One possibility is to use a time-centered implicit method. ${ }^{52,57}$ Another method that is being analyzed and implemented retains the staggered differencing between $\mathbf{V}$ and other fields in the time domain and uses linear implicit operators at each step. Terms associated with drift and dispersive waves are made numerically stable by non-self-adjoint differential operators, while the operator for MHD waves is unchanged. Taking the limit of zero electron mass, the staggered time-discrete form of the two-fluid model with drift effects is

$$
\begin{aligned}
m_{i} n^{j+1 / 2}\left(\frac{\Delta \mathbf{V}}{\Delta t}+\frac{1}{2} \mathbf{V}^{j} \cdot \nabla \Delta \mathbf{V}+\frac{1}{2} \Delta \mathbf{V} \cdot \nabla \mathbf{V}^{j}\right) \\
-\Delta t L^{j+1 / 2}(\Delta \mathbf{V})+\nabla \cdot \Pi_{i}(\Delta \mathbf{V})=\mathbf{J}^{j+1 / 2} \times \mathbf{B}^{j+1 / 2} \\
-m_{i} n^{j+1 / 2} \mathbf{V}^{j} \cdot \nabla \mathbf{V}^{j}-\nabla p^{j+1 / 2}-\nabla \cdot \Pi_{i}\left(\mathbf{V}^{j}\right), \\
\frac{\Delta n}{\Delta t}+\frac{1}{2} \mathbf{V}^{j+1} \cdot \nabla \Delta n=-\nabla \cdot\left(\mathbf{V}^{j+1} \cdot n^{j+1 / 2}\right), \\
\frac{3 n}{2}\left(\frac{\Delta T_{\alpha}}{\Delta t}+\frac{1}{2} \mathbf{V}_{\alpha}^{j+1} \cdot \nabla \Delta T_{\alpha}\right)+\frac{1}{2} \nabla \cdot \mathbf{q}_{\alpha}\left(\Delta T_{\alpha}\right)= \\
\quad-\frac{3 n}{2} \mathbf{V}_{\alpha}^{j+1} \cdot \nabla T_{\alpha}^{j+1 / 2}-n T_{\alpha}^{j+1 / 2} \nabla \cdot \mathbf{V}_{\alpha}^{j+1} \\
\quad-\nabla \cdot \mathbf{q}_{\alpha}\left(T_{\alpha}^{j+1 / 2}\right)+Q_{\alpha}^{j+1 / 2},
\end{aligned}
$$




$$
\begin{aligned}
\frac{\Delta \mathbf{B}}{\Delta t}+ & \frac{1}{2} \mathbf{V}^{j+1} \cdot \nabla \Delta \mathbf{B}+\frac{1}{2} \nabla \times \frac{1}{n e}\left(\mathbf{J}^{j+1 / 2} \times \Delta \mathbf{B}+\Delta \mathbf{J}\right. \\
& \left.\times \mathbf{B}^{j+1 / 2}\right)+\frac{1}{2} \nabla \times \eta \Delta \mathbf{J}=-\nabla \times\left[\frac { 1 } { n e } \left(\mathbf{J}^{j+1 / 2}\right.\right. \\
& \left.\left.\times \mathbf{B}^{j+1 / 2}-\nabla p_{e}\right)-\mathbf{V}^{j+1} \times \mathbf{B}^{j+1 / 2}+\eta \mathbf{J}^{j+1 / 2}\right],
\end{aligned}
$$

where superscripts show the time-level indices, and $\Delta$ indicates the change from a single step. The charge current density is directly related to magnetic field, $\mu_{0} \mathbf{J}=\nabla \times \mathbf{B}$, the number density is the same for the two species $(\alpha=\mathrm{i}, \mathrm{e})$, and $m_{i}$ is the ion mass. The ion flow velocity $\left(\mathbf{V}_{i}\right)$ is equivalent to the plasma flow (momentum carrying) velocity, while the electron flow velocity is $\mathbf{V}_{e}=\mathbf{V}-\mathbf{J} / n e$. The pressure $p$ (without subscript) is the sum of the electron and ion pressures, $n\left(T_{e}+T_{i}\right)$, and the differential operator $L$ in Eq. (76) is the linear MHD force operator,

$$
\begin{aligned}
L(\Delta \mathbf{V})= & \frac{1}{\mu_{0}}\{\nabla \times[\nabla \times(\Delta \mathbf{V} \times \mathbf{B})]\} \times \mathbf{B}+\mathbf{J} \times \nabla \times(\Delta \mathbf{V} \\
& \times \mathbf{B})+\nabla\left(\Delta \mathbf{V} \cdot \nabla p+\frac{5}{3} p \nabla \cdot \Delta \mathbf{V}\right) .
\end{aligned}
$$

Heating in Eq. (78) for each species appears as the term $Q_{\alpha}$, and $\eta$ is the electrical resistivity. The number density appearing in Eqs. (78) and (79) may be averaged from the $j+1 / 2$ and $j+3 / 2$ time levels, since both values are available at that point in the calculation. However, the $j+1$ level of electron flow in the electron temperature advance is not known until after the magnetic advance, so Eq. (78) may be solved first without the updated $\mathbf{V}_{\mathrm{e}}$ to predict $p_{\mathrm{e}}$ for Eq. (79), followed by a correction of electron temperature with the updated $\mathbf{V}_{\mathrm{e}}$.

Important drift effects from finite-sized gyro-orbits appear in Eqs. (76), (78), and (79). In the flow velocity evolution Eq. (76), they result from the gyro-viscous part of the traceless stress tensor, as described in Sec. II. The heat flux vectors $\mathbf{q}$ for separate species temperatures contain a term that directs heat perpendicular to both the magnetic field and the temperature gradient, $+2.5 p_{i}(e B)^{-1} \mathbf{b} \times \nabla T_{i}$ for ions and $-2.5 p_{e}(e B)^{-1} \mathbf{b} \times \nabla T_{e}$ for electrons. Equation (79) contains terms that are directly associated with drifts. Finite ion pressure leads to an imbalance between the Lorentz force and the electron pressure gradient, which causes magnetic perturbations to drift.

To ensure that the algorithm is numerically stable, the drift terms are implemented implicitly, as indicated in Eqs. (76)-(79). Unlike the MHD algorithm, ${ }^{28}$ the differential operators acting on the time-advanced fields are not selfadjoint. This has practical consequences, but once addressed, there is no additional computational penalty in making the advective terms implicit. The stability of the semi-implicit method for MHD with implicit advection has already been demonstrated, ${ }^{59}$ and numerical analysis shows that implicit advection is compatible with the two-fluid leapfrog algorithm.

The dispersion properties of the algorithm described above for the case of a uniform magnetic field are illustrated in Figs. 4 and 5. For both figures, length scales are measured

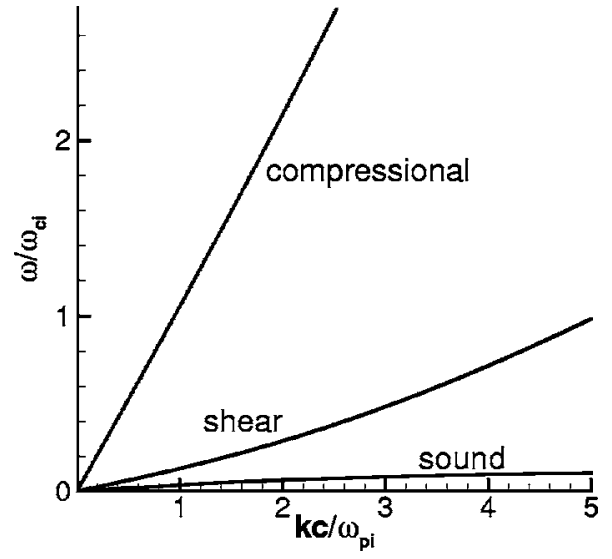

FIG. 4. Analytic dispersion relation for low-frequency waves propagating at an angle of $0.46 \pi$ from the uniform background magnetic field. The background pressure is such that $c_{s}^{2} / V_{A}^{2}=0.1$.

in terms of the ion skin depth $\Delta_{i}=c / \omega_{p i}$, and time is measured in terms of the inverse gyro-frequency $\Omega_{i}^{-1}$, so that the Alfvén speed is $V_{A}=\Omega_{i} \Delta_{i}$ and the Whistler frequency is $\omega_{W}$ $=k k_{\|}$. Figure 4 shows the analytic dispersion curves for the real part of the frequency for propagation at an angle $\theta$ $=0.46 \pi$ with respect to the magnetic field. Since there is no instability, the imaginary part of the frequency is zero. Figure 5 shows the real part of numerical frequency obtained from the algorithm of Eqs. (76)-(79) for the same angle of propagation with $\Delta t=1$ (in nondimensional units).

\section{BEYOND EXTENDED MHD CLOSURES}

As described in Sec. II, the extended MHD equations only capture the $O\left(\delta^{2}\right)$ FLR corrections to the resistive MHD model. This is often sufficient for the perpendicular motions. However, the parallel closures for fluid usually rely on some sort of collisional ordering for consistency. Since the parallel collisionality is often small in hot magnetized plasmas, these models may be inaccurate. Further, minority energetic ion species, such as beam or fusion $\alpha$ particles, may have large Larmor radii and therefore violate the assumptions of the

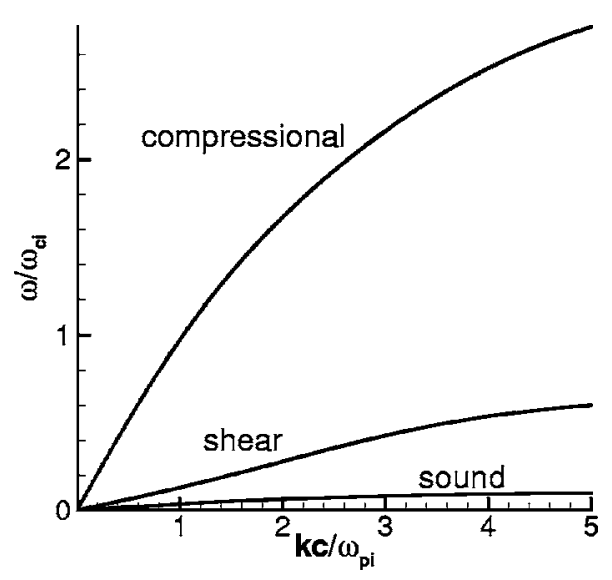

FIG. 5. Numerical dispersion relation for the implicit leapfrog algorithm for the physical parameters of Fig. 4 and $\Delta t=\Omega_{c i}^{-1}$. The dispersion introduced by the algorithm-ensuring numerical stability-is evident from comparison to Fig. 4. 
extended MHD model. Both of these cases require that a fuller kinetic theory be incorporated into the solution of the fluid equations.

In this section, we describe three approaches that have been used to obtain a more accurate solution of some aspects of the extended MHD model. In the first, described in Sec. $\mathrm{V}$ A, the effect of an energetic minority ion species is calculated using a fully kinetic model. The evolution of the minority species distribution function is solved numerically using the $\delta f$ method. $^{60}$ This calculation is subcycled within a time step of the advance of the extended MHD equations. The contribution of this species to the ion stress tensor is then computed at each point in space by taking the appropriate moments of the minority distribution function. This information is then used in the next time step of the fluid advance. In the second, described in Sec. V B, values for the parallel ion stress tensor and electron heat flux are obtained at each time step and at each point in space by computing the coefficients in a Chapman-Enskog-like ${ }^{61}$ expansion of the perturbed parallel distribution function. This involves numerical integration along perturbed field lines, and therefore captures nonlinear magnetic flutter effects. This information is then incorporated into an implicit solution of the fluid equations. The model is valid for all collisionality regimes. The third employs local models for the neoclassical stress. ${ }^{34}$ This is described in Sec. V C.

\section{A. Particle-in-cell closures for energetic particles}

The hybrid kinetic-MHD model ${ }^{60,62,63}$ incorporates the kinetic effects of a hot minority ion species that are not possible to simulate with fluid MHD simulation alone. Waveparticle interactions are known to strongly affect various MHD instabilities ${ }^{64}$ such as internal kink stabilization, generation of sawtooth and fishbone instabilities, and toroidal Alfvén eigenmodes.

To study the effects of a hot minority species on MHD modes in realistic geometry, we have implemented the $\delta f$ particle-in-cell (PIC) method ${ }^{60}$ for quadrilateral finite elements. We assume the minority ion density is small compared to the bulk plasma density $\left(n_{h} \ll n_{0}\right)$ (we will denote all minority species quantities by the subscript $h$ ), but that their pressure is comparable to the bulk plasma pressure $\left(\beta_{h}\right.$ $\left.\sim \beta_{0}\right)$. Under these assumptions, the only modification to the usual MHD equations is the addition of a hot particle pressure tensor to the fluid momentum equation. The kinetic particles are evolved in the MHD fields using the drift kinetic equations of motion. The perturbed pressure tensor is calculated from velocity moments of the kinetic particles using the $\delta f$ method.

In the limit of $n_{h} \ll n_{0}, \beta_{h} \sim \beta_{0}$, the only change to the MHD equations is the addition of the hot particle pressure term on the right hand side of the momentum equation,

$$
M n \frac{d \mathbf{V}}{d t}=\mathbf{J} \times \mathbf{B}-\nabla \cdot \Pi-\nabla \cdot \Pi_{h} .
$$

The equilibrium force balance condition is modified to include the equilibrium hot particle contribution,

$$
\mathbf{J} \times \mathbf{B}=\nabla \cdot \Pi+\nabla \cdot \Pi_{h},
$$

consistent with the assumption of $\beta_{h} \sim \beta_{0}$.

These equations are solved in a hybrid fashion by coupling PIC techniques for the hot minority species with the extended MHD code. The particles are evolved along drift kinetic orbits,

$$
\begin{aligned}
\frac{d \mathbf{x}}{d t}= & v_{\|} \mathbf{b}+\frac{M}{e B^{4}}\left(u^{2}+\frac{1}{2} v_{\perp}^{2}\right) \mathbf{B} \times \nabla \frac{B^{2}}{2}+\frac{\mathbf{E} \times \mathbf{B}}{B^{2}} \\
& +\frac{\mu_{0} M v_{\|}^{2}}{e B^{2}} \mathbf{J}_{\perp}, \\
M \frac{d v_{\|}}{d t} & =-\mathbf{b} \cdot(\mu \nabla B-e \mathbf{E}) .
\end{aligned}
$$

The adiabatic invariant, $\mu$, is taken as a parameter of the particle. We evolve an additional particle variable, the weight $\delta f$, which represents the deviation of the hot particle distribution function from its initial (equilibrium) value. The evolution equation for $\delta f$ along its characteristics is

$$
\frac{d \delta f}{d t}=-\delta \mathbf{v} \cdot \nabla f_{0}-e \mathbf{v}_{0} \cdot \mathbf{E} \frac{\partial f_{0}}{\partial \varepsilon},
$$

where $\mathbf{v}=\mathbf{v}_{0}+\delta \mathbf{v}$ and $f_{0}$ is the equilibrium hot particle distribution function.

At each time step, the perturbed hot pressure tensor is calculated by taking the $\delta f$ weighted second velocity moment of the particles in the usual PIC fashion,

$$
\Pi_{h}(\mathbf{x})=\int M\left(\mathbf{v}-\mathbf{V}_{h}\right)\left(\mathbf{v}-\mathbf{V}_{h}\right) \delta f(\mathbf{x}, \mathbf{v}) d^{3} \mathbf{v} .
$$

The hybrid kinetic MHD is used in several extended MHD codes. $^{28,49}$

As an example, we show results from a case that has been used as a benchmark with good agreement for the extended MHD codes NIMROD, ${ }^{28} \mathrm{M} 3 \mathrm{D},{ }^{49}$ and NOVA-K. ${ }^{62}$ The benchmark problem is the linear effect of the hot particles on the ideal $(m=1, n=1)$ internal kink mode in toroidal geometry. It is well known ${ }^{63}$ that a minority hot ion species can stabilize the ideal kink mode at low hot particle $\beta_{h}$ $\sim p_{h 0} / B_{0}^{2}$, and excite the fishbone branch at higher hot particle $\beta_{h}$. This has been demonstrated to good agreement in all three codes involved in the benchmark, as is shown in Fig. 6.

As the hot particle $\beta_{h}$ is increased from zero, the internal kink mode is stabilized and there is a corresponding increase in the real frequency of the $(m=1, n=1)$ mode. This stabilization mechanism has been interpreted as being due to the conservation of the third adiabatic invariant ${ }^{65}$ of trapped hot particles.

As $\beta_{h}$ is further increased, the fishbone mode emerges. This is precessional drift fishbone, ${ }^{62}$ not the diamagnetic fishbone ${ }^{66}$ since we advance only the single fluid equations for this problem. This precessional fishbone is a $m=1$ resonance of the precessional drift frequency of the trapped hot particles and the Alfvén continuum. This is evident in the 


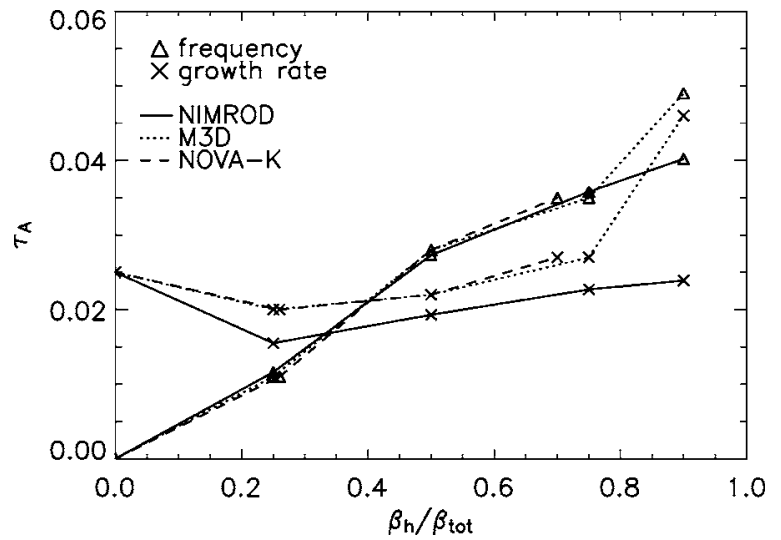

FIG. 6. Benchmark between three extended MHD codes using kinetic particles to describe the interaction between energetic minority ion species and the bulk fluid plasma for the case of an unstable $n=1$ MHD mode. As the energetic species contribution to the total pressure $\beta_{h}$ increases, the growth rate decreases (stabilization), but the real frequency (rotation rate) increases. The mode transitions from a kink to a fishbone.

approximate linear dependence of real frequency on the hot particle beta. The fishbone is also distinguished from the internal kink mode by its mode structure.

This is illustrated in Fig. 7, where we plot contours of the $n=1$ eigenmode of $V_{\phi}$ for $\beta_{h}=0.25$ (left) and $\beta_{h}=0.9$ (right). At $25 \%$, the flow is localized to the $q=1$ rational surface, similar to ideal $(1,1)$ internal kink. At $90 \%$, the fishbone eigenmode displays internal structure.

\section{B. Parallel kinetic closures}

In order to address the effects of long parallel collision lengths that characterize high-temperature magnetized plasmas, parallel kinetic closures may be implemented. These closures capture the kinetic physics of free-streaming and collisions in the form of expressions for the parallel heat flows, $\mathbf{q}_{\| s}=q_{\| s} \mathbf{b}$, and the parallel stress tensors, $\Pi_{\|_{s}}=(\mathbf{b b}$ $-\mathbf{I} / 3) \cdot \Pi_{s}$, which appear in the extended MHD equations.

The analytic theory underlying these closures is based on a Chapman-Enskog-like (CEL) approach, ${ }^{9,54}$ which ex-

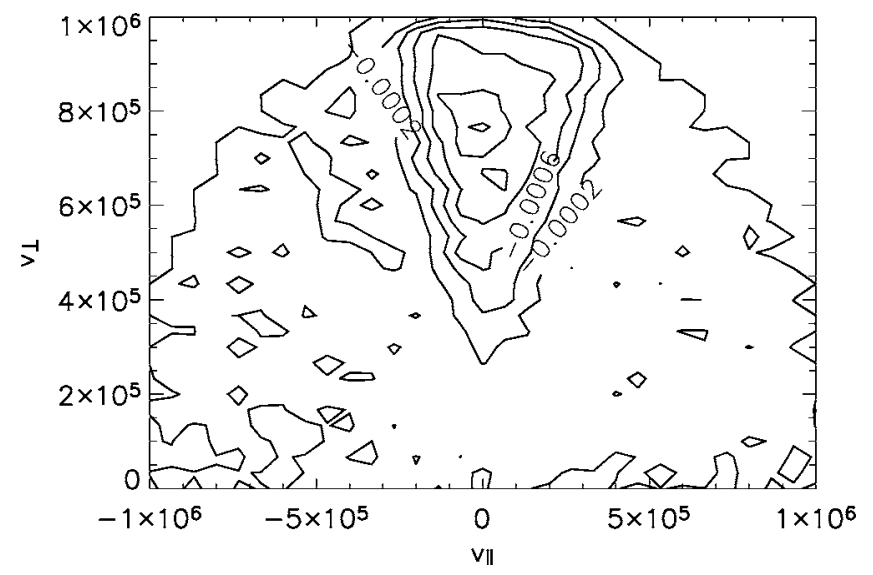

FIG. 7. Contours of the $n=1$ eigenmode of $V_{\phi}$ for $\beta_{h}=0.25$ (left) and $\beta_{h}$ $=0.9$ (right). At $25 \%$, the flow is localized to the $q=1$ rational surface, similar to ideal $(1,1)$ internal kink. At $90 \%$, the fishbone eigenmode displays internal structure.

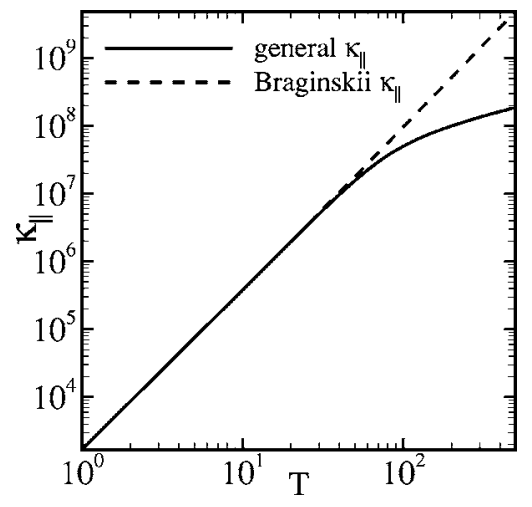

FIG. 8. Dashed line shows scaling of Braginskii $\kappa_{\|}$as the temperature varies from 1 to $500 \mathrm{eV}$. Solid line shows the effective $\kappa_{\|}$predicted by the general, parallel kinetic closure, $q_{\|}$. As the plasma becomes moderately collisional, $T>30 \mathrm{eV}$, the scaling of the Braginskii $\kappa_{\|}$is no longer correct and it becomes necessary to use the general form for $q_{\|}$.

presses the distribution function as the sum of a dynamic Maxwellian, $f_{M s}\left(n_{s}(\mathbf{x}, t), \mathbf{V}_{s}(\mathbf{x}, t), T_{s}(\mathbf{x}, t)\right)$, plus a kinetic distortion term, $F_{s}$. This notation implies that $f_{M s}$ evolves according to the solution for the lower order fluid moments, $n_{s}$, $\mathbf{V}_{s}$, and $T_{s}$. Over the course of a simulation, the gradient drives for $\mathbf{V}_{s}$ and $T_{s}$ give rise to $F_{s}$, whose moments provide closure for $q_{\| s}$ and $\Pi_{\|_{S}}$. In contrast to the $\delta f$ method (see Sec. $\mathrm{VA}$ ), which breaks down in long time-scale simulations when the perturbation becomes comparable to the constant, in the CEL approach the lowest-order distribution $F$ remains small compared to $f_{M}$ throughout a simulation. This feature permits application of these closures in simulations of resistive time-scale phenomena such as neoclassical tearing modes.

The derivation of parallel kinetic closures entails a solution of the drift kinetic equation for the lowest-order, gyrophase-independent portion of $F_{s}$. This solution allows for the maximal ordering between collisional and free-streaming effects. ${ }^{27,67,68}$ Taking the $q_{\| s}$ and $\Pi_{\| s}$ moments of $F_{s}$ results in the following statement of the closures as coupled integral equations:

$$
\begin{aligned}
q_{\| s}, \Pi_{\| s} \sim & \int_{0}^{\infty} d L^{\prime} K\left(L^{\prime}\right)\left\{\left.G\left(\mathbf{V}_{s}, T_{s}, q_{\| s}, \Pi_{\| s}\right)\right|_{+L^{\prime}}\right. \\
& \left.-\left.G\left(\mathbf{V}_{s}, T_{s}, q_{\| s}, \Pi_{\| s}\right)\right|_{-L^{\prime}}\right\} .
\end{aligned}
$$

Here $L^{\prime}$ denotes a coordinate along the magnetic field and the kernel, $K\left(L^{\prime}\right)$, contains the collisional and free-streaming information of the particles. The implementation of this solution involves integration along parallel trajectories characterized by the total magnetic field. The closures are therefore relevant in the important cases of stochastic magnetic fields and helical magnetic islands. In the collisional limit, the closures reproduce the familiar Braginskii parallel transport coefficients while in the moderately collisional to nearly collisionless limits, the closures capture free-streaming (phase mixing) and collisional effects and depend nonlocally on thermodynamic drives along the magnetic field (see Fig. 8). 


\section{Neoclassical closures}

As shown in Eq. (87), the closures in a near-collisionless regime are inherently nonlocal, because obtaining a value for the parallel heat flux or parallel stress at a grid point requires information along particle trajectories. On the time scales of fluid calculations, the electrons travel far along a field line, and it is useful to incorporate this physics without performing calculations on an electron transit time scale. Analytic closures that incorporate this nonlocality in toroidal geometry include neoclassical theory. ${ }^{33}$ This nonlocality manifests itself as the ability to calculate only the flux-surface-average of $\mathbf{B} \cdot \nabla \cdot \Pi$, and not local expressions.

The key step in these derivations ${ }^{33,69}$ is the calculation of the pressure anisotropy to relate the plasma flows to the stress. With this relationship, the flux-surface-averaged parallel components of the stress tensor and energy-weightedstress tensor can be calculated. Using a " $1 \times 1$ " matrix approximation, this relationship can be written as ${ }^{33}$

$$
\begin{aligned}
\left\langle\mathbf{B} \cdot \nabla \cdot \Pi_{\alpha}\right\rangle & =-\left\langle\left(p_{\|}-p_{\perp}\right)_{\alpha} \mathbf{b} \cdot \nabla B\right\rangle \\
& =n_{\alpha} m_{\alpha} \mu_{\alpha} \frac{\left\langle B^{2}\right\rangle \mathbf{b} \cdot \nabla B}{\left\langle(\mathbf{b} \cdot \nabla B)^{2}\right\rangle} u_{\theta}^{\alpha},
\end{aligned}
$$

where $u_{\theta}^{\alpha}=\mathbf{V}_{\alpha} \cdot \nabla \theta / \mathbf{B} \cdot \nabla \theta$. (In a " $2 \times 2$ " approximation, the energy-weighted stress tensor would be related to the flows and heat flux using a viscosity tensor.) The parallel stress tensor acts to damp flows in the poloidal direction as a result of variation in the magnitude of the magnetic field.

We seek a local expression to replace Eq. (88), but whose flux surface average is unchanged. Although the poloidal variation has been calculated ${ }^{70}$ for $\mathbf{B} \cdot \nabla \cdot \Pi$, the resulting expression has a singularity when inverting it to calculate the pressure anisotropy. This is due to the singularities at the turning points of the trapped particles. As an alternative, one can implement a local closure that has the correct fluxsurface-average properties, similar to what is done in deriving analytic expressions for neoclassical tearing modes. ${ }^{70} \mathrm{We}$ call these closures "heuristic closures" because the conversion of the nonlocal form to the local form is not rigorous, but useful. Using Eq. (88) as a guide, the heuristic closure is

$$
\nabla \cdot \Pi_{\alpha}=n_{\alpha} m_{\alpha} \mu_{\alpha}\left\langle B^{2}\right\rangle \frac{\mathbf{V}_{\alpha} \cdot \mathbf{e}_{\theta}}{\left(\mathbf{B} \cdot \mathbf{e}_{\theta}\right)^{2}} \mathbf{e}_{\theta} .
$$

This form has several favorable properties for numerical implementation. ${ }^{34}$ To show how this relationship captures the dominant physics for neoclassical tearing modes, we write the electron velocity $\mathbf{V}_{e}=\mathbf{V}_{i}-\mathbf{J} / n$ using force balance to lowest order, Eq. (34a),

$$
u_{\theta}^{e}=\frac{\mathbf{V}_{e} \cdot \nabla \theta}{\mathbf{B} \cdot \nabla \theta} \approx-\frac{\mathbf{J}^{-1}}{n e}\left(\frac{J_{\|}}{B}-\frac{1}{n e} \frac{I}{B^{2}} \frac{\partial p}{\partial \psi}\right),
$$

where $\mathrm{J}^{-1}$ is the inverse Jacobian and $I=R B_{T}$ is the toroidal flux function. The first term leads to neoclassical enhancement of the resistivity. The second term represents the bootstrap current. Early simulations of neoclassical tearing modes ${ }^{44,71}$ used just this term to represent the electron stress tensor.
The heuristic closure, Eq. (89), does not capture the toroidal drag induced by the perturbed particle orbits in the vicinity of the island. ${ }^{44}$ Because the island width scales as the square root of the perturbed field strength, this toroidal drag can be significant for large islands. A heuristic closure that has a toroidal component can be envisioned, but the viscosity coefficient would need to incorporate an island width scaling that results in no toroidal force in the limit of zero island width. The effect of linear growth rates on the viscosity coefficients has been investigated recently. ${ }^{67} \mathrm{Al}$ though this effect is likely very small in the Rutherford regime,${ }^{46}$ it is possible that a modified viscosity coefficient could be introduced. The goal of the closures to date has been to reproduce the analytic results that have been successfully used ${ }^{72-78}$ to analyze experimental results, and to allow the extension of analytic studies to include effects such as geometry and mode coupling.

Other analytic closures commonly used in the nearly collisionless regime include the Landau fluid closures. ${ }^{79,80} \mathrm{In}$ their simplest form, they are typically expressed as the Fourier transform of Eq. (87) [neglecting the magnetic geometry effects contained in the kernel, $\left.K\left(L^{\prime}\right)\right]$. In this form, the nonlocality enters formally only in terms of $\left|k_{\|}\right|$, which can be approximated easily if one is using a spectral code and an electrostatic approximation. The simplicity of this approach cannot be used for simulations of neoclassical tearing modes because of the need to include the effects of variations in the magnitude of the magnetic field along the field line, and the complexity of $\left|k_{\|}\right|$in three-dimensional magnetic geometry. Using a local approximation to the Landau fluid closures would be useful and consistent with the philosophy of the heuristic closure; however all neoclassical tearing mode calculations to date have used a Braginskii heat flux closure $^{44,71,74,77}$ The implementation of Landau-fluid models for the parallel heat flux closure would be an improvement to existing models and remains an active area of research.

\section{SUMMARY AND DISCUSSION}

Computational modeling of hot, magnetized plasmas requires extensions of the usual MHD model in order to capture the lowest order corrections to the dynamics in the small parameter $\delta=\rho_{i} / L$. The resulting equations contain a generalized Ohm's law, and closure expressions for higher order moments of the distribution function. While there is no general agreement on the specific form for these closures, it is essential to understand the fundamental dispersion and stability properties of the underlying equations, and their regions of validity, in order to design and implement efficient computational algorithms. This goal is facilitated by writing the fluid equations for the ion and electron species (with $\left.m_{e}=0\right)$ in nondimensional form. The parameters are $\varepsilon$ $=\omega / \Omega_{i}, \quad \xi=V_{0} / V_{t h i}, \quad \delta=V_{t h i} / \Omega_{i} L=\rho_{i} / L, \quad$ and $\nu / \Omega_{i}$. For a highly magnetized plasma $\delta \ll 1$, and important physical effects can be highlighted by the relative ordering of the remaining parameters with respect to $\delta$. In all cases, the electrons and ions flow as separate fluids.

Hall MHD appears in the ordering $\xi \sim 1 / \delta$ and $\varepsilon \sim 1$, which allows fast flows and large frequencies. In this order- 
ing the consistent Ohm's law contains the Whistler (Hall) terms, but ignores the diamagnetic contributions. Unbalanced forces appear at $O(1)$, so this model is appropriate for describing situations that are far from equilibrium, such as fast Z-pinches, gun plasma formation, and coronal mass ejections.

MHD appears in the ordering $\xi \sim 1$ and $\varepsilon \sim \delta$. MHD admits the possibility of fast flows $\left(V_{0} \sim V_{t h i}\right)$, but restricts the frequency to be low $\left(\omega \sim \delta \Omega_{i}\right)$. Unbalanced forces can appear at $O(\delta)$, so MHD is appropriate for describing situations that deviate moderately from equilibrium, such as spheromaks, RFPs, and possibly sawtooth crashes. FLR effects appear at $O\left(\delta^{2}\right)$ and are ignored in this model.

The drift ordering is restricted to slow flows $\left(V_{0}\right.$ $\left.\sim \delta V_{t h i}\right)$ and very low frequencies $\left(\omega \sim \delta^{2} \Omega_{i}\right)$. Unbalanced forces appear only at $O\left(\delta^{2}\right)$. This model is appropriate for systems that deviate only slightly and slowly from equilibrium, such as a hot plasma confined in a tokamak. If all FLR corrections are ignored, this ordering produces the transport model, in which all inertial effects are removed and the slow motions across the field are diffusive in nature. Retaining lowest order corrections $\left[O\left(\delta^{2}\right)\right.$ in the ion dynamics and $O(\delta)$ in the electron dynamics] yields dynamical equations that describe the ions and electrons as separate fluids.

In the drift ordering, the gyro-viscous force appears at the same order as the acceleration, and must be retained in the model. The neoclassical parallel viscous force also enters at the same order, and should be retained. This ordering allows a cancellation to occur between the advection of the ion diamagnetic drift velocity and the ion gyro-viscous stress that yields the equations of the standard drift model. The exact form of this cancellation depends on the specific problem under consideration.

Drift models often serve as the starting point for analytical investigations of tokamak plasmas. However, they have several disadvantages for building general computational models. In the first place, the uncertainties inherent in the gyro-viscous cancellation, which is an essential part of the drift model, may be comparable with other uncertainties introduced by discretization of the equations for computational implementation, or with artificial dissipation required for highly nonlinear calculations. Secondly, the dependent velocity in the drift model does not carry the fluid momentum. In and of itself this is not a severe disadvantage, but it does complicate the implementation of boundary conditions and interpretation of the results, as the dependent variables are one step removed from the fundamental physical quantities. Third, the drift model is only valid under the restrictions of the drift ordering, which only allows small $\left[O\left(\delta^{2}\right)\right]$ deviations from exact force balance. Thus it is applicable primarily to tokamaks, and then only in the very early stages of departure from equilibrium conditions. It may not be applicable to studies of other magnetoplasma configurations, or of highly nonlinear dynamics. For these reasons, we have chosen to implement the primitive form of the extended MHD equations, and to use accurate and efficient numerical methods to provide the resulting cancellations.

The primitive extended MHD equations contain highfrequency dispersive waves that may nonetheless affect the low-frequency fluid response. The presence of these parasitic waves dictates the use of implicit numerical methods that allow time steps to exceed $10^{4,5}$ times the explicit CFL limit. Semi-implicit methods have been useful in this regard. When coupled with a high order finite-element representation, these methods have proven useful for obtaining accurate solutions for the low-frequency dynamics of a variety of magnetoplasma configurations.

The extended MHD equations only capture the lowest order corrections in the Larmor radius to the usual MHD model. Higher order corrections are needed to describe the effects of energetic minority ion species and low collisionality parallel transport on the fundamental plasma dynamics. Energetic species can be studied by using a hybrid-kinetic model to integrate the perturbed minority ion distribution function along perturbed particle orbits in the evolving fields described by the fluid model. The effect of this species on the bulk evolution is captured by computing the hot particle stress tensor by numerical integration of the appropriate moments of the perturbed distribution function.

Parallel kinetic transport effects are computed by a moment expansion of the parallel plasma kinetic equation. Numerical implementation requires accurate and efficient integration of the expansion functions along the perturbed magnetic field lines. Resulting expressions for the parallel viscous stress and parallel heat flux are valid for all collisionalities. They agree with the Braginskii values for large collision frequency, and provide a natural flux limitation as the collision frequency becomes small. Values for the parallel transport are then incorporated pointwise into the fluid model.

Neoclassical effects account for the interaction between passing and trapped particles in a toroidal plasma, and are difficult to quantify as local expressions. Most theories are formulated as flux surface averages, while for computation local expressions are needed. Heuristic local expressions have been proposed that retain the same flux surface average as the more rigorous expressions, while conserving energy and increasing entropy. They can account for bootstrap current, neoclassical resistivity, and enhanced polarization current. When used with extended MHD models that treat anisotropic heat flux with great accuracy, they are able to reproduce theoretically predicted thresholds for the onset of the neoclassical tearing mode. ${ }^{34}$

Because of the extreme complexity of the underlying kinetic problem, fluid models are likely to form the basis for advanced computational modeling of magnetized plasmas for the foreseeable future. A major research thrust is to extend these models even further, for example to capture the effects of radio frequency wave deposition on global dynamics, to incorporate more accurate models for the cold plasma near the edge of a tokamak, and to provide a more accurate description of the interaction of the hot plasma with its material boundary. These efforts require integration of extended fluid models with other equally complex physics models. This is a short-term challenge for theoretical and computational plasma physics, and for computer science. 


\section{ACKNOWLEDGMENTS}

The authors wish to thank Drs. Elena Belova, Steven Jardin, Thomas Gianakon, Alan Glasser, Jesus Ramos, and Linda Sugiyama for enlightening discussions on this topic. We are especially indebted to Professor James Callen for guidance and instruction in extended fluid models. We also gratefully acknowledge the pioneering work of Drs. Guo Yong Fu, Wonchull Park, Scott E. Parker, and Henry R. Strauss in the field of multilevel plasma modeling. We value our collaboration with them as part of the Center for Extended MHD Modeling, under the Scientific Discover through Advanced Computing (SciDAC) initiative. One of us (D.S.) wishes to acknowledge the contributions of Professor John Killeen to the development of computational physics as a rigorous scientific discipline, and his influence on the work described herein. This work was performed under Grants No. DE-FC02-02ER54668, No. DE-FC0204ER54798, No. DE-FC03-02ER54666, No. DE-FG0395ER54309, and No. DE-FG02-04ER54799 with the U. S. Department of Energy.

${ }^{1}$ H. Strauss, Phys. Fluids 19, 134 (1976).

${ }^{2}$ H. Strauss, Phys. Fluids 20, 1354 (1977).

${ }^{3}$ R. Izzo, D. A. Monticello, W. Park, H. Strauss, J. Manickam, R. Grimm, and K. McGuire, Phys. Fluids 26, 2240 (1983).

${ }^{4}$ H. Strauss, Nucl. Fusion 23, 649 (1984).

${ }^{5}$ M. N. Rosenbluth and A. Simon, Phys. Fluids 8, 1300 (1965).

${ }^{6}$ R. D. Hazeltine and J. D. Meiss, Phys. Rep. 121, 1 (1985).

${ }^{7}$ C. T. Hsu, R. D. Hazeltine, and P. J. Morrison, Phys. Fluids 29, 1480 (1986).

${ }^{8}$ F. L. Hinton and C. W. Horton, Jr., Phys. Fluids 14, 116 (1971).

${ }^{9}$ Z. Chang and J. D. Callen, Phys. Fluids B 4, 1766 (1992).

${ }^{10}$ A. I. Smolyakov, Can. J. Phys. 76, 321 (1998).

${ }^{11}$ E. V. Belova, Phys. Plasmas 8, 3936 (2001).

${ }^{12}$ L. E. Sugiyama and W. Park, Phys. Plasmas 7, 4644 (2000).

${ }^{13}$ R. D. Hazeltine and J. D. Meiss, Plasma Confinement (Addison-Wesley, Redwood City, CA, 1992).

${ }^{14}$ I. Lindemuth and J. Killeen, J. Comput. Phys. 13, 181 (1973).

${ }^{15}$ D. D. Schnack, J. A. Dibiase, J. Killeen, and D. C. Robinson, "Linear and non-linear calculation of the tearing mode in reversed field pinches," in Pulsed High Beta Plasmas (Pergamon Press, Oxford, 1976), p. 283.

${ }^{16}$ D. D. Schnack and J. Killeen, "Linear and non-linear calculations of the tearing mode," in Theoretical and Computational Plasma Physics (IAEA, Vienna, 1978), p. 337.

${ }^{17}$ D. D. Schnack and J. Killeen, Nucl. Fusion 19, 877 (1979).

${ }^{18}$ J. Killeen, A. I. Shestakov, and D. D. Schnack, "Linear and non-linear calculations of resistive magnetohydrodynamic instabilities," in Computing Methods in Applied Science and Engineering, edited by R. Glowinski and J. Lions (North Holland, Amsterdam, 1980), p. 305.

${ }^{19}$ D. D. Schnack and J. Killeen, J. Comput. Phys. 35, 110 (1980).

${ }^{20}$ D. D. Schnack, D. C. Baxter, and E. J. Caramana, J. Comput. Phys. 55, 485 (1984).

${ }^{21}$ D. S. Harned and D. D. Schnack, J. Comput. Phys. 65, 57 (1986).

${ }^{22}$ D. D. Schnack, Z. Mikic, D. S. Harned, E. J. Caramana, and D. C. Barnes, J. Comput. Phys. 70, 330 (1987).

${ }^{23}$ D. D. Schnack, Z. Mikic, D. C. Barnes, and G. Van Hoven, Comput. Phys. Commun. 59, 21 (1990).

${ }^{24}$ D. D. Schnack, I. Lottati, Z. Mikic, and P. Satyanarayana, J. Comput. Phys. 140, 71 (1998).

${ }^{25}$ R. Lionello, Z. Mikic, and D. D. Schnack, J. Comput. Phys. 140, 172 (1998).

${ }^{26}$ A. H. Glasser, C. R. Sovinec, R. A. Nebel, T. A. Gianakon, S. J. Plimpton, M. S. Chu, D. D. Schnack, and the NIMROD Team, Plasma Phys. Controlled Fusion 41, A747 (1999).

${ }^{27}$ C. R. Sovinec, T. A. Gianakon, E. Held, S. E. Kruger, D. D. Schnack, and the NIMROD Team, Phys. Plasmas 10, 1727 (2003).
${ }^{28}$ C. R. Sovinec, A. H. Glasser, T. A. Gianakon, D. C. Barnes, R. A. Nebel, S. E. Kruger, D. D. Schnack, S. J. Plimpton, A. Tarditi, M. S. Chu, and the NIMROD Team, J. Comput. Phys. 195, 355 (2004).

${ }^{29}$ L. D. Landau and E. M. Lifshitz, Electrodynamics of Continuous Media (Pergamon Press, Oxford, 1960).

${ }^{30}$ K. V. Roberts and J. B. Taylor, Phys. Rev. Lett. 8, 197 (1962).

${ }^{31}$ A. Hasegawa and L. Chen, Phys. Fluids 19, 1924 (1976).

${ }^{32}$ S. I. Braginskii, "Transport processes in a plasma," in Reviews of Plasma Physics, edited by M. A. Leontovich (Consultants Bureau, New York, 1965), Vol. 1, p. 205.

${ }^{33}$ S. P. Hirshman and D. J. Sigmar, Nucl. Fusion 21, 1079 (1981).

${ }^{34}$ T. A. Gianakon, S. E. Kruger, and C. C. Hegna, Phys. Plasmas 9, 536 (2002).

${ }^{35}$ A. B. Mikhailovskii and V. S. Tsypin, Beitr. Plasmaphys. 24, 335 (1984).

${ }^{36}$ P. J. Catto and A. N. Simakov, Phys. Plasmas 11, 90 (2004).

${ }^{37}$ J. J. Ramos, Phys. Plasmas 12, 052102 (2005).

${ }^{38}$ T. H. Stix, Waves in Plasmas (Springer-Verlag, New York, 1992).

${ }^{39}$ H. P. Furth, J. Killeen, and M. N. Rosenbluth, Phys. Fluids 6, 459 (1963).

${ }^{40}$ M. Kwizak and A. Robert, Mon. Weather Rev. 99, 32 (1971).

${ }^{41}$ W. Park and D. A. Monticello, Nucl. Fusion 30, 2413 (1990).

${ }^{42}$ E. J. Caramana, J. Comput. Phys. 96, 484 (1991).

${ }^{43}$ K. Lerbinger and J. F. Luciani, J. Comput. Phys. 97, 444 (1991).

${ }^{44}$ A. M. Popov, V. S. Chan, M. S. Chu, Y. Q. Liu, B. W. Rice, and A. D. Turnbull, Phys. Plasmas 8, 3605 (2001).

${ }^{45}$ R. W. Freund, G. H. Golub, and N. M. Nachtigal, Acta Numerica pp. 57-100 (1991).

${ }^{46}$ P. H. Rutherford, Phys. Fluids 16, 1903 (1973).

${ }^{47}$ X. S. Li and J. W. Demmel, ACM Trans. Math. Softw. 29, 110 (2003), for example.

${ }^{48}$ L. A. Charlton, J. A. Holmes, V. E. Lynch, and B. A. Carreras, J. Comput. Phys. 86, 270 (1990).

${ }^{49}$ W. Park, E. V. Belova, G. Y. Fu, X. Z. Tang, H. R. Strauss, and L. E. Sugiyama, Phys. Plasmas 6, 1796 (1999).

${ }^{50}$ L. E. Sugiyama, W. Park, H. R. Strauss, S. R. Hudson, D. Stutman, and X.-Z. Tang, Nucl. Fusion 41, 739 (2004).

${ }^{51}$ G. Strang and G. J. Fix, An Analysis of the Finite Element Method (Prentice-Hall, Englewood Cliffs, NJ, 1973).

${ }^{52}$ S. C. Jardin and J. A. Breslau, Phys. Plasmas 12, 056101 (2005).

${ }^{53}$ M. D. Gunzburger, "Mathematical aspects of finite element methods for incompressible viscous flows," in Finite Element Theory and Application: Proceedings of the ICASE Finite Element Theory and Application Workshop (Hampton, Virginia, 28-30 July, 1986) edited by D. L. Dwoyer, M. Y. Hussaini, and R. G. Voigt (Springer-Verlag, New York, 1988), pp 124150.

${ }^{54}$ E. D. Held, J. D. Callen, C. C. Hegna, C. R. Sovinec, T. A. Gianakon, and S. E. Kruger, Phys. Plasmas 11, 2419 (2004).

${ }^{55}$ R. Lionello, Z. Mikić, and J. A. Linker, J. Comput. Phys. 152, 346 (1999).

${ }^{56}$ L. Chacón, D. A. Knoll, and J. M. Finn, J. Comput. Phys. 178, 15 (2002).

${ }^{57}$ L. Chacón and D. A. Knoll, J. Comput. Phys. 188, 573 (2003); D. C. Barnes, Bull. Am. Phys. Soc. 49, 183 (2004).

${ }^{58}$ D. S. Harned and Z. Mikić, J. Comput. Phys. 83, 1 (1989).

${ }^{59}$ C. R. Sovinec and the NIMROD Team, Bull. Am. Phys. Soc. 48, 115 (2003).

${ }^{60}$ S. E. Parker and W. W. Lee, Phys. Fluids B 5, 77 (1993).

${ }^{61} \mathrm{~S}$. Chapman and T. G. Cowling, The Mathematical Theory of NonUniform Gases (Cambridge University Press, Cambridge, 1939).

${ }^{62}$ C. Z. Cheng, J. Geophys. Res. 96, 21 (1991).

${ }^{63}$ C. Z. Cheng and J. R. Robinson, J. Geophys. Res. 104, 413 (1999).

${ }^{64}$ W. Park, S. Parker, H. Biglari et al., Phys. Fluids B 4, 2033 (1992).

${ }^{65}$ F. Porcelli, Plasma Phys. Controlled Fusion 33, 1601 (1991).

${ }^{66}$ B. Coppi and F. Porcelli, Phys. Rev. Lett. 57, 2272 (1986).

${ }^{67}$ E. D. Held, J. D. Callen, and C. C. Hegna, Phys. Plasmas 10, 3933 (2003); E. D. Held, ibid. 10, 4708 (2003).

${ }^{68}$ K. C. Shaing, Phys. Plasmas 12, 2511 (2005).

${ }^{69}$ J. P. Wang and J. D. Callen, Phys. Fluids B 5, 3207 (1993).

${ }^{70}$ C. C. Hegna, Phys. Plasmas 6, 3980 (1999).

${ }^{71}$ T. A. Gianakon, C. C. Hegna, and J. D. Callen, Phys. Plasmas 3, 4637 (1996).

${ }^{72}$ K. C. Shaing, C. C. Hegna, J. D. Callen, and W. A. Houlberg, Nucl. Fusion 43, 258 (2003).

${ }^{73}$ R. LaHaye, L. Lao, E. Strait, and T. Taylor, Nucl. Fusion 37, 397 (1997).

${ }^{74}$ D. A. Gates, B. Lloyd, A. W. Morris, G. McArdle, M. R. O'Brien, M. Valovic, C. D. Warrick, and H. R. Wilson, Nucl. Fusion 37, 1593 (1997).

${ }^{75}$ O. Sauter, R. J. La Haye, Z. Chang et al., Phys. Plasmas 5, 1654 (1997). 
${ }^{76}$ S. Gunter, A. Gude, M. Maraschek, Q. Yu, and the ASDEX Upgrade Team, Plasma Phys. Controlled Fusion 41, 767 (1999).

${ }^{77}$ H. Lutjens, J. F. Luciani, A. Bondeson, and X. Garbet, Phys. Plasmas 9, 219 (2002).
${ }^{78}$ Q. Yu, S. Gunter, G. Giruzzi, K. Lackner, and M. Zabiego, Phys. Plasmas 7, $312(2000)$

${ }^{79}$ G. W. Hammett and F. W. Perkins, Phys. Rev. Lett. 64, 3019 (1990).

${ }^{80}$ P. B. Snyder and G. W. Hammett, Phys. Plasmas 8, 744 (2001). 